\title{
PRINCIPAL HODGE REPRESENTATIONS
}

\author{
C. ROBLES
}

\begin{abstract}
We study Hodge representations of absolutely simple $\mathbb{Q}$-algebraic groups with Hodge numbers $\mathbf{h}=(1,1, \ldots, 1)$. For those groups that are not of type $\mathrm{A}$, we give a classification of the $\mathbb{R}$-irreducible representations; a similar classification for type A does not seem possible.
\end{abstract}

\section{INTRODUCTION}

This paper is a study of Hodge structures with Hodge numbers $\mathbf{h}=(1,1, \ldots, 1)$. Examples of such Hodge structures include the cohomology group $H^{1}(X, \mathbb{Q})$ of an elliptic curve, the cohomology group $H^{3}(X, \mathbb{Q})$ of a mirror quintic variety [3], and the weight component $W_{6} H^{6}(X, \mathbb{Q})$ of the cohomology group of a general fibre of a family of quasi-projective 6 -folds studied by Dettweiler and Reiter [2,6]. More precisely, the article addresses the question: what are the Hodge representations (§2.1) with Hodge numbers $\mathbf{h}=(1,1, \ldots, 1)$ ? To simplify exposition, we call such representations 'principal,' cf. Remark 2.6. The principal Hodge representations are necessarily of Calabi-Yau type; Calabi-Yau Hodge representations of (absolutely) simple Hodge groups are classified in [8, §B]. I emphasize that the classification of principal Hodge representations is essentially a representation theoretic question; I will not directly address the Hodge theory of complex algebraic varieties.

Motivation. Very roughly, the classification question is motived by the question: Does a Hodge domain admit a distinguished realization as a Mumford-Tate domain. A more precise statement of the question, and its relation to this work, is given in Section 2.2?

Contents. The main results of this paper are characterizations of the principal Hodge representations $(V, \varphi)$ of a $\mathbb{Q}$-algebraic, absolutely simple ${ }^{[\mathrm{i}]}$ group $G$ in the case that (i) the vector space $V_{\mathbb{R}}$ is an irreducible $G_{\mathbb{R}}$-module, and (ii) the Lie algebra $\mathfrak{g}_{\mathbb{C}} \neq \mathfrak{s l}_{r+1} \mathbb{C}$. The principal Hodge representations of symplectic groups (type C) are identified in Theorem 3.1, those of orthogonal groups (types B and D) are identified in Theorems 4.1 and 4.3, and those of the exceptional groups of type $G_{2}$ are identified in Theorem 5.2. The exceptional groups of types $E_{r}$ and $F_{4}$ admit no such principal Hodge representations (Corollary 2.15 and Theorem 5.1). (For the exceptional groups, we may drop the assumption (ii) that $V_{\mathbb{R}}$ is irreducible.)

Date: July 20, 2021.

2010 Mathematics Subject Classification. 20G05, 58A14.

Key words and phrases. Hodge group, Hodge representation, Hodge structure, Mumford-Tate group.

Robles is partially supported by NSF DMS-1006353.

$\left.{ }^{[i}\right]_{G}$ is absolutely simple if $G_{\mathbb{C}}$ is simple. 
I am unable to give a similar characterization in the case that $\mathfrak{g}_{\mathbb{C}}=\mathfrak{s l}_{r+1} \mathbb{C}$ (type A). A comparison of the theorems above with the results and examples of $\S 6$ suggest that no such characterization is available in this case: given a representation $\pi: G \rightarrow \operatorname{Aut}(V, Q)$, the circle $\varphi$ giving $\pi$ the structure of a principal Hodge representation is essentially unique (if it exists) for types B, C, D and G; in contrast, the circle is far from unique in type A, cf. §6.5. Nonetheless, we may make some general observations (cf. §6.1); for example, if $G$ admits a principal Hodge representation (with $V_{\mathbb{R}}$ not necessarily irreducible), then the rank $r$ is necessarily odd (Lemma 6.4). The rank one, three and five cases are worked out in $\S 6.2,6.3$ and 6.4 , respectively, and examples of rank seven and nine are considered in $\S 6.5$.

Acknowledgements. I thank P. Griffiths for bringing the classification question to my attention.

\section{Hodge RePresentations}

2.1. Definition. Let $V$ be a rational vector space, $w \in \mathbb{Z}$, and let $Q=V \times V \rightarrow \mathbb{Q}$ be a nondegenerate bilinear form satisfying $Q(u, v)=(-1)^{w} Q(v, u)$, for all $u, v \in V$. A polarized Hodge structure of weight $w$ on $V$ is given by a nonconstant homomorphism $\phi$ : $S^{1} \rightarrow \operatorname{Aut}\left(V_{\mathbb{R}}, Q\right)$ of $\mathbb{R}$-algebraic groups with the properties that $\phi(-1)=(-1)^{w} \mathbb{1}$, and $Q(v, \phi(\mathbf{i}) \bar{v})>0$ for all $0 \neq v \in V_{\mathbb{C}}$. (Here, $\mathbf{i}=\sqrt{-1}$.) The associated Hodge decomposition $V_{\mathbb{C}}=\oplus_{p+q=w} V^{p, q}$ is given by $V^{p, q}=\left\{v \in V_{\mathbb{C}} \mid \phi(z) v=z^{p-q} v, \forall z \in S^{1}\right\}$. The Hodge numbers $\mathbf{h}=\left(h^{p, q}\right)$ of $\phi$ are $h^{p, q}=\operatorname{dim}_{\mathbb{C}} V^{p, q}$.

Let $G$ be a $\mathbb{Q}$-algebraic group, and let $\pi: G \rightarrow \operatorname{Aut}(V, Q)$ be a homomorphism of $\mathbb{Q}$ algebraic groups, such that $\pi_{*}: \mathfrak{g}_{\mathbb{C}} \rightarrow \operatorname{End}(V, Q)$ is injective. Let $\varphi: S^{1} \rightarrow G_{\mathbb{R}}$ be a nonconstant homomorphism of $\mathbb{R}$-algebraic groups. Then $(V, Q, \pi, \varphi)$ is a Hodge representation of $G$ if $\pi \circ \varphi$ is a $Q$-polarized Hodge structure on $V$ of weight $w$. Any $\mathbb{Q}$-algebraic group admitting a Hodge representation is a Hodge group. A Hodge group $G$ is necessarily reductive [4, (I.B.6)], and $G_{\mathbb{R}}$ contains a compact, maximal torus $T \supset \varphi\left(S^{1}\right)$; that is, $\operatorname{dim}_{\mathbb{R}}(T)=\operatorname{rank}\left(G_{\mathbb{R}}\right)$, cf. [4, (IV.A.2)].

In general, the bilinear form $Q$ and representation $\pi$ will be dropped from the notation, and the Hodge representation will be denoted by $(V, \varphi)$.

2.2. Context. Hodge representations were introduced by Green, Griffiths and Kerr in [4] to determine: (i) which reductive, $\mathbb{Q}$-algebraic groups $G$ admit the structure of a MumfordTate group, and (ii) what one can say about various realizations of $G$ as a MumfordTate group, and the associated Mumford-Tate domains (which generalize period domains). Mumford-Tate groups are the symmetry groups of Hodge theory: the Mumford-Tate group $G_{\phi} \subset \mathcal{G}=\operatorname{Aut}(V, Q)$ of a polarized Hodge structure $(V, Q, \phi)$ is precisely the $\mathbb{Q}$-algebraic group preserving the Hodge tensors [4, (I.B.1)]. Moreover, $\phi\left(S^{1}\right) \subset G_{\phi}(\mathbb{R})$, so that $(V, Q, \phi)$ is a Hodge representation of $G_{\phi}$, and the Mumford-Tate group is a Hodge group.

Mumford-Tate domains arise as follows. Let $F^{p}=\oplus_{q \geq p} V^{q, w-q}$ denote the Hodge filtration of $V_{\mathbb{C}}=V \otimes_{\mathbb{Q}} \mathbb{C}$ induced by the Hodge structure $\phi$. Define Hodge numbers $f^{p}=\operatorname{dim}_{\mathbb{C}} F^{p}$ and $\mathbf{f}=\left(f^{\bullet}\right)$. Then $F^{\bullet}$ is an element of the isotropic flag variety $\operatorname{Flag}_{\mathbf{f}}^{Q}\left(V_{\mathbb{C}}\right)$, and the latter is a $\mathcal{G}_{\mathbb{C}}=\operatorname{Aut}\left(V_{\mathbb{C}}, Q\right)$-homogeneous manifold. (If the weight $w$ is even, then $\mathcal{G}_{\mathbb{C}}=\operatorname{SO}\left(V_{\mathbb{C}}\right)$ is an orthogonal group; if $w$ is odd, then $\mathcal{G}_{\mathbb{C}}=\operatorname{Sp}\left(V_{\mathbb{C}}\right)$ is a symplectic group.) The period 
domain $\mathcal{D} \subset \operatorname{Flag}_{\mathbf{f}}^{Q}\left(V_{\mathbb{C}}\right)$ is the $\mathcal{G}_{\mathbb{R}}=\operatorname{Aut}\left(V_{\mathbb{R}}, Q\right)$-orbit of $F^{\bullet}$. The Mumford-Tate domain $D \subset \mathcal{D}$ is the $G_{\phi}(\mathbb{R})$-orbit of $F^{\bullet}$.

As a $G_{\phi}(\mathbb{R})$-homogeneous manifold $D \simeq G_{\phi}(\mathbb{R}) / R$; here $R$ is the centralizer of the circle $\phi\left(S^{1}\right)$ and contains the compact, maximal torus $T$, cf. [4, II.A]. The homogeneous manifold $G_{\phi}(\mathbb{R}) / R$ is a Hodge domain. One may see from this discussion that one subtlety that arises in the subject is the fact that a Hodge domain will admit various realizations as a Mumford-Tate domain (and with inequivalent $G_{\phi}(\mathbb{R})$-homogeneous complex structures). One would like to know if a given Hodge domain admits a distinguished realization as a Mumford-Tate domain. ${ }^{[i]}$ The classification of principal Hodge representations is motivated by this question.

A Hodge representation $(V, \phi)$ of $G$ is principal if and only if $f^{p+1}=f^{p}-1$, cf. Definition 2.5. Equivalently, the period domain $\mathcal{D}=\operatorname{Flag}_{\mathbf{f}}^{Q}\left(V_{\mathbb{C}}\right)$ is a full flag variety, and the horizontal distribution (known as Griffiths' transversality or the infinitesimal period relation) on the period domain $\mathcal{D}$ is bracket-generating. In this case, the centralizer $R$ of the circle $\phi\left(S^{1}\right)$ is the torus $T$, cf. Remark 2.18. So the principal Hodge representation yields a distinguished realization of the Hodge domain $G_{\phi}(\mathbb{R}) / T$ as a Mumford-Tate domain $D \subset \mathcal{D}$.

2.3. The grading element $\mathrm{T}_{\varphi}$. Fix a Hodge representation $(V, \varphi)$. To the circle $\varphi\left(S^{1}\right)$ is naturally associated a semisimple $\mathrm{T}_{\varphi} \in \mathbf{i} \mathfrak{g}_{\mathbb{R}}$. What follows is a terse review of $\mathrm{T}_{\varphi}$; see $[8, \S 2.3]$ for details. The semisimple $\mathrm{T}_{\varphi}$ has the property that the $\mathrm{T}_{\varphi}$-eigenvalues of $V_{\mathbb{C}}$ are rational numbers (in fact, elements of $\frac{1}{2} \mathbb{Z}$ ), and the $\mathrm{T}_{\varphi}$-eigenspace decomposition

$$
V_{\mathbb{C}}=\bigoplus_{\ell \in \mathbb{Q}} V_{\ell}, \quad V_{\ell} \stackrel{\text { dfn }}{=}\left\{v \in V_{\mathbb{C}} \mid \mathrm{T}_{\varphi}(v)=\ell v\right\}
$$

is the Hodge decomposition: that is, $V^{p, q}=V_{\ell}$, where $\ell=(p-q) / 2 \in \frac{1}{2} \mathbb{Z}$. Additionally, the $\mathrm{T}_{\varphi}$-eigenvalues of $\mathfrak{g}_{\mathbb{C}}$ are integers, and the $\mathrm{T}_{\varphi}$-eigenspace decomposition

$$
\mathfrak{g}_{\mathbb{C}}=\bigoplus_{\ell \in \mathbb{Z}} \mathfrak{g}_{\ell}, \quad \mathfrak{g}_{\ell} \stackrel{\mathrm{dfn}}{=}\left\{\zeta \in \mathfrak{g}_{\mathbb{C}} \mid\left[\mathrm{T}^{\ell}, \zeta\right]=\ell \zeta\right\}
$$

is the Hodge decomposition associated to the weight zero Hodge structure Ad $\circ \pi$ on $\mathfrak{g}$ : that is, $\mathfrak{g}^{\ell,-\ell}=\mathfrak{g}_{\ell}$. Moreover,

$$
\mathfrak{g}_{\ell}\left(V_{q}\right) \subset V_{q+\ell} \text { and }\left[\mathfrak{g}_{k}, \mathfrak{g}_{\ell}\right] \subset \mathfrak{g}_{k+\ell} .
$$

In particular, the Hodge filtration $F^{\bullet}$ of $V_{\mathbb{C}}$, defined by $F^{p}=V_{\geq p}$, is stabilized by the parabolic subalgebra

$$
\mathfrak{p} \stackrel{\text { dfn }}{=} \mathfrak{g}_{\geq 0}
$$

of $\mathfrak{g}_{\mathbb{C}}$.

Fix a compact maximal torus $T \subset G_{\mathbb{R}}$ containing the circle $\varphi\left(S^{1}\right)$. Let $\mathfrak{t} \subset \mathfrak{g}_{\mathbb{R}}$ and $\mathfrak{t}_{\mathbb{C}} \subset \mathfrak{g}_{\mathbb{C}}$ denote the associated Lie algebras. Then $\mathrm{T}_{\varphi} \in$ it. Let $\Delta=\Delta\left(\mathfrak{g}_{\mathbb{C}}, \mathfrak{t}_{\mathbb{C}}\right) \subset \mathfrak{t}_{\mathbb{C}}^{*}$ denote

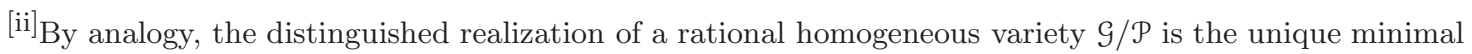
homogeneous embedding $\mathcal{G} / \mathcal{P} \hookrightarrow \mathbb{P} \mathcal{V}$. The Plücker embedding of the Grassmannian $\operatorname{Gr}\left(k, \mathbb{C}^{n}\right) \simeq \operatorname{SL}\left(\mathbb{C}^{n}\right) / \mathcal{P}_{k}$ is an example.
} 
the roots of $\mathfrak{t}_{\mathbb{C}}$. Let $\Lambda_{\mathrm{rt}} \subset \Lambda_{\mathrm{wt}} \subset \mathfrak{t}_{\mathbb{C}}^{*}$ denote the root and weight lattices. The connected real groups $G_{\mathbb{R}}$ with Lie algebra $\mathfrak{g}_{\mathbb{R}}$ are indexed by sub-lattices $\Lambda_{\mathrm{rt}} \subset \Lambda \subset \Lambda_{\mathrm{wt}}$. The torus is

$$
T=\mathfrak{t} / \Lambda^{*}, \quad \text { where } \Lambda^{*} \stackrel{\text { dfn }}{=} \operatorname{Hom}(\Lambda, 2 \pi \mathbf{i} \mathbb{Z}),
$$

and the weights $\Lambda\left(V_{\mathbb{C}}\right)$ of $V_{\mathbb{C}}$ are contained in $\Lambda$. (Conversely, if $U$ is an irreducible $\mathfrak{g}_{\mathbb{C}^{-}}$ module of highest weight $\mu \in \Lambda$, then $U$ is a $G_{\mathbb{C}^{-}}$module.) Any $\mathrm{T} \in \operatorname{Hom}\left(\Lambda, \frac{1}{2} \mathbb{Z}\right)$ determines a circle $\varphi: S^{1} \rightarrow T$ such that $\mathrm{T}_{\varphi}=\mathrm{T}$.

Notice that $\mathfrak{t}_{\mathbb{C}} \subset \mathfrak{g}_{0} \subset \mathfrak{p}$; so we may select a Borel subalgebra $\mathfrak{b}$ so that $\mathfrak{t}_{\mathbb{C}} \subset \mathfrak{b} \subset \mathfrak{p}$. Define positive roots by

$$
\Delta^{+} \stackrel{\text { dfn }}{=} \Delta(\mathfrak{b})=\left\{\alpha \in \Delta \mid \mathfrak{g}^{\alpha} \subset \mathfrak{b}\right\} .
$$

Let $\Sigma=\left\{\sigma_{1}, \ldots, \sigma_{r}\right\}$ denote the simple roots of $\Delta^{+}$, and let $\left\{\mathrm{T}^{1}, \ldots, \mathrm{T}^{r}\right\} \subset \operatorname{Hom}\left(\Lambda_{\mathrm{rt}}, \mathbb{Z}\right) \subset \mathfrak{t}_{\mathbb{C}}$ denote the dual basis of $\mathfrak{t}_{\mathbb{C}}$; that is, $\sigma_{i}\left(\mathrm{~T}^{j}\right)=\delta_{i}^{j}$. The lattice $\operatorname{Hom}\left(\Lambda_{\mathrm{rt}}, \mathbb{Z}\right)$ is the set of grading elements. The fact that the $\mathrm{T}_{\varphi}$-eigenvalues of $\mathfrak{g}_{\mathbb{C}}$ are integers implies $\mathrm{T}_{\varphi} \in \operatorname{Hom}\left(\Lambda_{\mathrm{rt}}, \mathbb{Z}\right)$; that is, $\mathrm{T}_{\varphi}$ is a grading element. Therefore, $\mathrm{T}_{\varphi}$ is necessarily of the form $n_{i} \mathrm{~T}^{i}$ for some $n_{i} \in \mathbb{Z}$. The condition $\mathfrak{g}^{\sigma_{i}} \subset \mathfrak{b} \subset \mathfrak{p}=\mathfrak{g}_{\geq 0}$ is equivalent to

$$
\sigma_{i}\left(\mathrm{~T}_{\varphi}\right)=n_{i} \geq 0
$$

for all $1 \leq i \leq r$.

2.4. Real, complex and quaternionic representations. Suppose that $V_{\mathbb{R}}$ is an irreducible $G_{\mathbb{R}}$-module. Then there exists an irreducible $G_{\mathbb{C}}$-module $U$ such that one of the following holds.

○ As a $G_{\mathbb{R}}-$ module $U$ is real: $V_{\mathbb{C}}=U \simeq U^{*}$.

○ As a $G_{\mathbb{R}}$-module $U$ is quaternionic: $V_{\mathbb{C}}=U \oplus U^{*}$ and $U \simeq U^{*}$.

○ As a $G_{\mathbb{R}}-$ module $U$ is complex: $V_{\mathbb{C}}=U \oplus U^{*}$ and $U \not 千 U^{*}$.

Notice that $U$ is complex if and only if $U$ is not self-dual. The real and quaternionic cases are distinguished as follows. Define

$$
\mathrm{T}^{\mathrm{cpt}} \stackrel{\text { dfn }}{=} 2 \sum_{n_{i} \in 2 \mathbb{Z}} \mathrm{T}^{i}
$$

Let $\mu \in \Lambda(U) \subset \Lambda$ be the highest weight of $U$. If $U \simeq U^{*}$, then

$$
U \text { is real (resp., quaternionic) if and only if } \mu\left(\mathrm{T}^{\mathrm{cpt}}\right) \text { is even (resp., odd), }
$$

cf. [4, (IV.E.4)].

\subsection{Principal Hodge representations. Let}

$$
m \stackrel{\text { dfn }}{=} \max \left\{q \in \mathbb{Q} \mid V_{q} \neq 0\right\} \in \frac{1}{2} \mathbb{Z} .
$$

Then the $\mathrm{T}_{\varphi}$-eigenspace decomposition of $V_{\mathbb{C}}$ is

$$
V_{\mathbb{C}}=V_{m} \oplus V_{m-1} \oplus \cdots \oplus V_{1-m} \oplus V_{-m},
$$

and the Hodge numbers are $\mathbf{h}=\left(h_{m}, h_{m-1}, \ldots, h_{1-m}, h_{-m}\right)$, where $h_{\ell}=\operatorname{dim}_{\mathbb{C}} V_{\ell}$.

Definition 2.5. The Hodge representation $(V, \varphi)$ is principal if the Hodge numbers are $\mathbf{h}=(1,1, \ldots, 1,1)$; that is $h_{\ell}=1$ for all $-m \leq \ell \leq m$. 
Remark 2.6. The Hodge representation $(V, \varphi)$ is principle if and only if $\mathrm{T}_{\varphi}$ is the monosemisimple element of a principal $\mathfrak{s l}_{2} \mathbb{C} \subset \mathfrak{s l}\left(V_{\mathbb{C}}\right)$. See [7].

If $(V, \varphi)$ is principal, then it is necessarily the case that

$$
\begin{aligned}
& \text { the } \mathrm{T}_{\varphi} \text {-eigenvalues of } V_{\mathbb{C}} \text { are multiplicity-free, } \\
& \text { and } m=\frac{1}{2}\left(\operatorname{dim}_{\mathbb{C}} V_{\mathbb{C}}-1\right) .
\end{aligned}
$$

The eigenvalues are determined by the weights $\Lambda\left(V_{\mathbb{C}}\right) \subset \mathfrak{t}_{\mathbb{C}}^{*}$ of $V_{\mathbb{C}}$. Precisely, the $\mathrm{T}_{\varphi^{-}}$ eigenvalues of $V_{\mathbb{C}}$ are $\left\{\lambda\left(\mathrm{T}_{\varphi}\right) \mid \lambda \in \Lambda\left(V_{\mathbb{C}}\right)\right\}$. In particular, $(V, \varphi)$ is principal if and only if

$$
\left\{\lambda\left(\mathrm{T}_{\varphi}\right) \mid \lambda \in \Lambda\left(V_{\mathbb{C}}\right)\right\}=\{m, m-1, m-2, \ldots, 2-m, 1-m,-m\}
$$

and (2.8) holds.

Note that the $\mathrm{T}_{\varphi}$-eigenvalues of $V_{\mathbb{C}}$ are multiplicity-free only if

$$
V_{\mathbb{C}} \text { is weight multiplicity-free. }
$$

Recall that $\Lambda\left(U^{*}\right)=-\Lambda(U)$. So, if $V_{\mathbb{C}}=U \oplus U^{*}$, then $\Lambda\left(V_{\mathbb{C}}\right)=\Lambda(U) \cup-\Lambda(U)$. The necessary condition (2.10) implies

$$
\begin{gathered}
\text { The } \mathfrak{g}_{\mathbb{C}} \text {-module } U \text { is either real or complex. } \\
\text { If } U \text { is complex, then } \Lambda(U) \cap-\Lambda(U)=\emptyset \text {. }
\end{gathered}
$$

2.6. Multiplicity-free representations. Suppose that $(V, \varphi)$ is a principal Hodge representation, and that $V^{0} \subset V_{\mathbb{R}}$ is an irreducible $G_{\mathbb{R}}$-submodule. Let $U$ be the corresponding irreducible $\mathfrak{g}_{\mathbb{C}}$-module, cf. $§ 2.4$. By (2.10), it is necessarily the case that

$$
U \text { is weight multiplicity-free. }
$$

The weight multiplicity-free representations have been classified by [5, Theorem 4.6.3], in the case that $\mathfrak{g}_{\mathbb{C}}$ is simple. Let $\omega_{1}, \ldots, \omega_{r}$ denote the fundamental weights of $\mathfrak{g}_{\mathbb{C}}$.

Theorem 2.14. Let $\mathfrak{g}_{\mathbb{C}}$ be a simple, complex Lie group. The irreducible, weight multiplicityfree representations $U$ of $\mathfrak{g}_{\mathbb{C}}$, with highest weight $\mu$, are as follows:

(a) If $\mathfrak{g}_{\mathbb{C}}=\mathfrak{s l}_{r+1} \mathbb{C}$, then $U$ is one of $\bigwedge^{k} \mathbb{C}^{r+1}$, and $\mu=\omega_{k}$; $\operatorname{Sym}^{a} \mathbb{C}^{r+1}$, and $\mu=a \omega_{1}$; or $\operatorname{Sym}^{a}\left(\mathbb{C}^{r+1}\right)^{*}$, and $\mu=a \omega_{r}$.

(b) If $\mathfrak{g}_{\mathbb{C}}=\mathfrak{s o}_{2 r+1} \mathbb{C}$, then $U$ is either the standard representation $\mathbb{C}^{2 r+1}$, with $\mu=\omega_{1}$; or the spin representation, with $\mu=\omega_{r}$.

(c) If $\mathfrak{g}_{\mathbb{C}}=\mathfrak{s p}_{2 r} \mathbb{C}$, then either $U$ is the standard representation $\mathbb{C}^{2 r}$, with $\mu=\omega_{1}$; or $r \in\{2,3\}$ and $U \subset \wedge^{r} \mathbb{C}^{2 r}$, with $\mu=\omega_{r}$.

(d) If $\mathfrak{g}_{\mathbb{C}}=\mathfrak{s o}_{2 r} \mathbb{C}$, then $U$ is either the standard representation $\mathbb{C}^{2 r}$, with $\mu=\omega_{1}$; or one of the spin representations, with $\mu=\omega_{r-1}, \omega_{r}$.

(e) If $\mathfrak{g}_{\mathbb{C}}$ is the exceptional Lie algebra $\mathfrak{e}_{6}(\mathbb{C})$, then $\mu=\omega_{1}, \omega_{6}$ and $\operatorname{dim}_{\mathbb{C}} U=27$; if $\mathfrak{g}_{\mathbb{C}}=$ $\mathfrak{e}_{7}(\mathbb{C})$, then $\mu=\omega_{7}$ and $\operatorname{dim}_{\mathbb{C}} U=56$. If $\mathfrak{g}_{\mathbb{C}}$ is the exceptional Lie algebra $\mathfrak{g}_{2}(\mathbb{C})$, then $U=\mathbb{C}^{7}$ and $\mu=\omega_{1}$. There are no weight multiplicity-free representations for the exceptional Lie algebras $\mathfrak{e}_{8}$ and $\mathfrak{f}_{4}$.

From (2.13) and Theorem 2.14(e) we obtain the following. 
Corollary 2.15. The exceptional Lie groups of type $E_{8}$ and $F_{4}$ admit no principal Hodge representations.

2.7. Weights. It will be helpful to review some well-known properties of the weights $\Lambda(U)$ of the irreducible $\mathfrak{g}_{\mathbb{C}}$-module $U$. Assume $\mathfrak{g}_{\mathbb{C}}$ is semi-simple, and let $\mu$ denote the highest weight of $U$. Given any weight $\lambda \in \Lambda(U)$, there exists an (ordered) sequence $\left\{\sigma_{i_{1}}, \ldots, \sigma_{i_{\ell}}\right\}$ of simple roots such that

(i) $\lambda=\mu-\left(\sigma_{i_{1}}+\cdots+\sigma_{i_{\ell}}\right)$, and

(ii) $\mu-\left(\sigma_{i_{1}}+\cdots+\sigma_{i_{j}}\right) \in \Lambda(U)$, for all $1 \leq j \leq \ell$.

By (i), any weight $\lambda$ of $U$ is of the form $\mu-\lambda^{i} \sigma_{i}$, for some $0 \leq \lambda^{i} \in \mathbb{Z}$. So, (2.2) implies $\mu\left(\mathrm{T}_{\varphi}\right)$ is the largest eigenvalue of $U$ :

$$
\lambda\left(\mathrm{T}_{\varphi}\right) \leq \mu\left(\mathrm{T}_{\varphi}\right) \text { for all } \lambda \in \Lambda(U) .
$$

If $U^{\mu} \subset U$ is the highest weight line, then the weight space of $\lambda=\mu-\left(\sigma_{i_{1}}+\cdots+\sigma_{i_{\ell}}\right)$ is $U^{\lambda}=\mathfrak{g}^{-\sigma_{i_{\ell}}} \cdots \mathfrak{g}^{-\sigma_{i_{1}}} \cdot U^{\mu}$. In particular, since $\pi_{*}: \mathfrak{g}_{\mathbb{C}} \rightarrow \operatorname{End}(V, Q)$ is injective, for each $1 \leq i \leq r$, there exists $\lambda \in \Lambda(U)$ such that $\lambda-\sigma_{i} \in \Lambda(U)$. Therefore, both $\lambda\left(\mathrm{T}_{\varphi}\right)$ and $\lambda\left(\mathrm{T}_{\varphi}\right)-n_{i}$ are eigenvalues of $U$. In particular, $\mathrm{T}_{\varphi}$-eigenvalues of $V_{\mathbb{C}}$ are multiplicity-free only if $n_{i} \neq 0$ for all $1 \leq i \leq r$. By $(2.2), n_{i} \geq 0$. Therefore, if the Hodge representation $(V, \varphi)$ is principal, it is necessarily the case that

$$
n_{i}>0
$$

for all $1 \leq i \leq r$.

Remark 2.18. It follows from this discussion and (2.1) that the stabilizer $\mathfrak{p}=\mathfrak{g}_{\geq 0}$ of the Hodge filtration $F^{\bullet}$ in $\mathfrak{g}_{\mathbb{C}}$ is the Borel $\mathfrak{b}$. In particular, the Hodge domain is $G_{\phi}(\mathbb{R}) / T$, with $T$ a compact, maximal torus.

Let $\mu^{*}$ denote the highest weight of $U^{*}$. Swapping $U$ and $U^{*}$ if necessary, we may assume that

$$
\mu\left(\mathrm{T}_{\varphi}\right) \leq \mu^{*}\left(\mathrm{~T}_{\varphi}\right)
$$

Then (2.9) and (2.16) yield

$$
m=\mu\left(\mathrm{T}_{\varphi}\right)
$$

Define

$$
m^{*} \stackrel{\text { dfn }}{=} \mu^{*}\left(\mathrm{~T}_{\varphi}\right) \leq m .
$$

By (2.9), any two $\mathrm{T}_{\varphi}$-eigenvalues of $V_{\mathbb{C}}$ differ by an integer; therefore

$$
0 \leq m-m^{*} \in \mathbb{Z} .
$$

If $U$ is complex, so that $V_{\mathbb{C}}=U \oplus U^{*}$ then (2.7) and (2.19) force

$$
m^{*}<m \text {. }
$$

The notation

$$
\left(\lambda^{1} \cdots \lambda^{r}\right) \stackrel{\text { dfn }}{=} \lambda=\mu-\lambda^{i} \sigma_{i}
$$

will be convenient. For example, $\mu$ is denoted $(0 \cdots 0)$. Set

$$
|\lambda|=\sum \lambda^{i}
$$


The weights $\{\lambda \in \Lambda(U):|\lambda|=1\}$ may be described very easily: let $\mu=\mu^{i} \omega_{i}$; then, $\mu-\sigma_{i}$ is a weight of $U$ if and only if $\mu^{i} \neq 0$, cf. [1, Proposition 3.2.5]. If $\mathfrak{g}_{\mathbb{C}}$ is simple, and $U$ is weight multiplicity-free, then $\mu=p \omega_{i}$, by Theorem 2.14. Therefore, the two largest $\mathrm{T}_{\varphi}$-eigenvalues of $U$ are $m=\mu\left(\mathrm{T}_{\varphi}\right)$ and $m-n_{i}=\left(\mu-\sigma_{i}\right)\left(\mathrm{T}_{\varphi}\right)$. The following lemma is a consequence of this discussion and equations (2.7) and (2.9).

Lemma 2.22. Assume that $\mathfrak{g}_{\mathbb{C}}$ is simple. Suppose that $(V, \varphi)$ is a principal Hodge representation of $G$, and that $V_{\mathbb{R}}$ is an irreducible $G_{\mathbb{R}}$-submodule. Let $U$ be the associated irreducible, weight multiplicity-free $\mathfrak{g}_{\mathbb{C}}$-module (cf. §2.4) of highest weight $\mu=p \omega_{i}$, and satisfying (2.19).

(a) If $U$ is real $\left(V_{\mathbb{C}}=U\right)$, then $n_{i}=1$.

(b) Suppose $U$ is complex $\left(V_{\mathbb{C}}=U \oplus U^{*}\right.$ and $\left.U \neq U^{*}\right)$. Then $0<m-m^{*} \in \mathbb{Z}$. Define $1 \leq i^{*} \leq r$ by $\mu^{*}=p \omega_{i^{*}}$. Then $m^{*}=m-1$ if and only if $n_{i}>1$; and $m^{*}=m-1$ and $n_{i^{*}}=1$ if and only if $n_{i}>2$.

\section{Symplectic Hodge groups}

Theorem 3.1. Let $G$ be a Hodge group with complex Lie algebra $\mathfrak{g}_{\mathbb{C}}=\mathfrak{s p}_{2 r} \mathbb{C}$. Assume that $(V, \varphi)$ is a Hodge representation with the property that $V_{\mathbb{R}}$ is an irreducible $G_{\mathbb{R}}$-module. Let $\mathrm{T}_{\varphi}$ be the associated grading element (\$2.3), and assume the normalization (2.2) holds. Then the Hodge representation is principal if and only if one of the following holds:

(a) $V_{\mathbb{C}}=\mathbb{C}^{2 r}$ is the standard representation, and $\mathrm{T}_{\varphi}=\mathrm{T}^{1}+\cdots+\mathrm{T}^{r}$;

(b) $r=2, V_{\mathbb{C}} \subset \wedge^{2} \mathbb{C}^{4}$ is the irreducible $G_{\mathbb{C}}$-module of highest weight $\omega_{2}$, and $\mathrm{T}=\mathrm{T}^{1}+\mathrm{T}^{2}$;

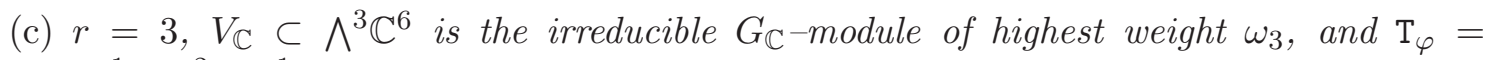
$3 \mathrm{~T}^{1}+\mathrm{T}^{2}+\mathrm{T}^{1}$.

Proof. The representations of $G_{\mathbb{C}}$ are self-dual. So the representation $U$ associated to $V_{\mathbb{R}}$ (cf. §2.4) is either real or quaternionic. By $(2.11)$, if $(V, \varphi)$ is principal, then $U$ is necessarily real, so that $V_{\mathbb{C}}=U$. The condition $(2.13)$ and Theorem $2.14(\mathrm{c})$ restrict our attention to the case that $U$ is one of the the following fundamental representations: either $U_{\omega_{1}}=\mathbb{C}^{2 r}$ of highest weight

$$
\omega_{1}=\left(\sigma_{1}+\cdots+\sigma_{r-1}+\frac{1}{2} \sigma_{r}\right),
$$

for arbitrary $r$; or $U_{\omega_{r}}=\bigwedge^{r} \mathbb{C}^{2 r}$ of highest weight

$$
\begin{aligned}
\omega_{2}=\sigma_{1}+\sigma_{2} & \text { if } r=2, \\
\omega_{3}=\sigma_{1}+2 \sigma_{2}+\frac{3}{2} \sigma_{3} & \text { if } r=3 .
\end{aligned}
$$

(A) Let's begin with the case that $\mu=\omega_{1}$. The weights of $\mathbb{C}^{2 r}$ are

$$
\begin{aligned}
\Lambda\left(\mathbb{C}^{2 r}\right)= & \left\{\omega_{1}\right\} \cup\left\{\omega_{1}-\left(\sigma_{1}+\cdots+\sigma_{i}\right) \mid 1 \leq i \leq r\right\} \\
& \cup\left\{\omega-\left(\sigma_{1}+\cdots+\sigma_{i-1}\right)-2\left(\sigma_{i}+\cdots+\sigma_{r-1}\right)-\sigma_{r} \mid 1 \leq i \leq r-1\right\} .
\end{aligned}
$$

It is straightforward to check that $\mathrm{T}_{\varphi}=\mathrm{T}^{1}+\cdots+\mathrm{T}^{r}$ is the unique grading element satisfying the normalization (2.2), and yielding the consecutive, multiplicity-free eigenvalues (2.9). 
(B) Next, consider the case that $r=2$ and $\mu=\omega_{2}$. Here $\operatorname{dim}_{\mathbb{C}} U=5$ and the weights of $U$ are

$$
\begin{aligned}
\Lambda(U) & =\left\{ \pm\left(\sigma_{1}+\sigma_{2}\right), \pm \sigma_{1}, 0\right\} \\
& =\left\{\omega_{2}, \omega_{2}-\sigma_{2}, \omega_{2}-\left(\sigma_{1}+\sigma_{2}\right), \omega_{2}-\left(2 \sigma_{1}+\sigma_{2}\right), \omega_{2}-2\left(\sigma_{1}+\sigma_{2}\right)\right\} .
\end{aligned}
$$

It is clear that $\mathrm{T}=\mathrm{T}^{1}+\mathrm{T}^{2}$ is the unique element satisfying the normalization (2.2), and yielding the consecutive, multiplicity-free eigenvalues (2.9).

(C) Finally, suppose that $r=3$ and $\mu=\omega_{3}$. Then $\operatorname{dim}_{\mathbb{C}} U=14$, and the weights of $U$ are

$$
\begin{aligned}
\Lambda(U)= & \{(000),(001),(011),(021),(022),(111),(121), \\
& (122),(132),(221),(222),(232),(242),(243)\} .
\end{aligned}
$$

Above, we utilize the notation $\left(\lambda^{1} \lambda^{2} \lambda^{3}\right)=\omega_{3}-\left(\lambda^{1} \sigma_{1}+\lambda^{2} \sigma_{2}+\lambda^{3} \sigma_{3}\right)$ introduced in $\S 2.7$. In particular, the weights of $U$ include $\left\{\omega_{3}, \omega_{3}-\sigma_{3}, \omega_{3}-\left(\sigma_{2}+\sigma_{3}\right)\right\}$. Since the remaining weights are all of the form $\omega_{3}-\left(a \sigma_{1}+b \sigma_{2}+c \sigma_{3}\right)$, with $0 \leq a, b, c \in \mathbb{Z}$ and $a+b+c \geq 3$, this forces $1=n_{3}=n_{2}$.

The conditions (2.8) and (2.20) yield $13 / 2=\mu\left(\mathrm{T}_{\varphi}\right)=\mu\left(n_{1} \mathrm{~T}^{1}+\mathrm{T}^{2}+\mathrm{T}^{3}\right)$, so that $n_{1}=3$. Thus, the grading element is necessarily of the form given in (c). Given $\mathrm{T}_{\varphi}=3 \mathrm{~T}^{1}+\mathrm{T}^{2}+\mathrm{T}^{3}$, is straight-forward to compute $\Lambda(U)\left(\mathrm{T}_{\varphi}\right)=\{13 / 2,11 / 2, \ldots,-11 / 2,-13 / 2\}$. Thus $(2.9)$ holds.

Finally, observe that in each of the cases (A), (B) and (C) above, $(2.3)$ yields $\mathrm{T}^{\mathrm{cpt}}=0$, so that $\mu\left(\mathrm{T}^{\mathrm{cpt}}\right)=0$ and $V_{\mathbb{C}}=U$ is real by $(2.4)$, as required.

\section{Orthogonal Hodge groups}

Theorem 4.1. Let $G$ be a $\mathbb{Q}$-algebraic group with complex Lie algebra $\mathfrak{g}_{\mathbb{C}}=\mathfrak{s o}_{2 r+1} \mathbb{C}$. Assume that $(V, \varphi)$ is a Hodge representation of $G$ with the property that $V_{\mathbb{R}}$ is an irreducible $G_{\mathbb{R}}$-module. Let $\mathrm{T}_{\varphi}$ be the associated grading element ( $(2.3)$, and assume the normalization (2.2) holds. Then the Hodge representation is principal if and only if one of the following holds:

(a) $V_{\mathbb{C}}=\mathbb{C}^{2 r+1}$ is the standard representation, and $\mathrm{T}_{\varphi}=\mathrm{T}^{1}+\cdots+\mathrm{T}^{r}$;

(b) $V_{\mathbb{C}}=U_{\omega_{r}}$ is the spin representation,

$$
\mathrm{T}_{\varphi}=2^{r-2} \mathrm{~T}^{1}+2^{r-3} \mathrm{~T}^{2}+2^{r-4} \mathrm{~T}^{3}+\cdots+2 \mathrm{~T}^{r-2}+\mathrm{T}^{r-1}+\mathrm{T}^{r},
$$

and $(r-2)(r-1) \in 4 \mathbb{Z}$.

Theorem 4.3. Let $G$ be a Hodge group with complex Lie algebra $\mathfrak{g}_{\mathbb{C}}=\mathfrak{s o}_{2 r} \mathbb{C}$. Assume that $(V, \varphi)$ is a Hodge representation with the property that $V_{\mathbb{R}}$ is an irreducible $G_{\mathbb{R}}$-module. Let $\mathrm{T}_{\varphi}$ be the associated grading element (\$2.3), and assume the normalization (2.2) holds. Then the Hodge representation is principal if and only if one of the following holds:

(a) $r \geq 4$ is even, $V_{\mathbb{C}}=U$ is a spin representation $\left(\mu=\omega_{r-1}, \omega_{r}\right)$,

$$
\mathrm{T}_{\varphi}=2^{r-3} \mathrm{~T}^{1}+2^{r-4} \mathrm{~T}^{2}+2^{r-5} \mathrm{~T}^{3}+\cdots+2 \mathrm{~T}^{r-3}+\mathrm{T}^{r-2}+\mathrm{T}^{r-1}+\mathrm{T}^{r},
$$

and $(r-3)(r-2) \in 4 \mathbb{Z}$; 
(b) $r \geq 5$ is odd, $V_{\mathbb{C}}=U \oplus U^{*}$, where $U \neq U^{*}$ is a spin representation $\left(\mu=\omega_{r-1}, \omega_{r}\right)$ and $\mathrm{T}_{\varphi}$ is one of

$$
\begin{aligned}
& \mathrm{T}_{\varphi}=2^{r-2} \mathrm{~T}^{1}+2^{r-3} \mathrm{~T}^{2}+\cdots+4 \mathrm{~T}^{r-3}+2 \mathrm{~T}^{r-2}+\mathrm{T}^{r-1}+3 \mathrm{~T}^{r}, \\
& \mathrm{~T}_{\varphi}=2^{r-2} \mathrm{~T}^{1}+2^{r-3} \mathrm{~T}^{2}+\cdots+4 \mathrm{~T}^{r-3}+2 \mathrm{~T}^{r-2}+3 \mathrm{~T}^{r-1}+\mathrm{T}^{r} .
\end{aligned}
$$

Proof of Theorem 4.1. Let $U$ be the irreducible $\mathfrak{s o}_{2 r+1} \mathbb{C}$-module associated to $V_{\mathbb{R}}$ as in $§ 2.4$. All representations of $\mathfrak{s o}_{2 r+1} \mathbb{C}$ are self-dual; therefore, if $(V, \varphi)$ is principal, it is necessarily the case that $U$ is a real representation of $G_{\mathbb{R}}$, by $(2.11)$, and $V_{\mathbb{C}}=U$. By (2.13), $U$ is weight multiplicity-free. The irreducible, multiplicity-free representations of $\mathfrak{s o}_{2 r+1} \mathbb{C}$ are given by Theorem 2.14(b): either $U=\mathbb{C}^{2 r+1}$ is the standard representation, with highest weight

$$
\omega_{1}=\left(\sigma_{1}+\sigma_{2}+\cdots+\sigma_{r}\right)
$$

or $U$ is the spin representation of highest weight

$$
\omega_{r}=\frac{1}{2}\left(\sigma_{1}+2 \sigma_{2}+\cdots+r \sigma_{r}\right) .
$$

We consider each case below.

The standard representation. Suppose that $U=\mathbb{C}^{2 r+1}$ is the standard representation. The weights of $U$ are

$$
\begin{aligned}
\Lambda\left(V_{\mathbb{C}}\right)= & \left\{\omega_{1}\right\} \cup\left\{\omega_{1}-\left(\sigma_{1}+\cdots+\sigma_{i}\right) \mid 1 \leq i \leq r\right\} \\
& \cup\left\{\omega_{1}-\left(\sigma_{1}+\cdots+\sigma_{i-1}\right)+2\left(\sigma_{i}+\cdots+\sigma_{r}\right) \mid 1 \leq i \leq r\right\} .
\end{aligned}
$$

It is straight forward to confirm that $\mathrm{T}_{\varphi}=\mathrm{T}^{1}+\cdots+\mathrm{T}^{r}$ is the unique grading element satisfying the normalization (2.2) and such that the $\mathrm{T}_{\varphi}$-eigenvalues of $U$ satisfy (2.8) and (2.9).

In particular, $\mathrm{T}^{\mathrm{cpt}}=0$, by $(2.3)$, so that $U$ is real, by $(2.4)$, as required by $(2.11)$.

The spin representation: preliminaries. Suppose that $U$ is the spin representation. Then $\mu=\omega_{r}$. The weights of $U$ are parameterized by

(4.6a) $\mathcal{L}(U)=\left\{\lambda=\left(\lambda^{1}, \ldots, \lambda^{r}\right) \in \mathbb{Z}^{r} \mid \lambda^{1} \in\{0,1\}\right.$, and $\left.\lambda^{i}-\lambda^{i-1} \in\{0,1\}, \forall 1<i \leq r\right\} ;$

specifically,

$$
\Lambda(U)=\left\{\omega_{r}-\lambda^{i} \sigma_{i} \mid \lambda \in \mathcal{L}(U)\right\} .
$$

It will be convenient to define (i) a filtration $\{0\}=\mathcal{F}^{0} \subset \mathcal{F}^{1} \subset \mathcal{F}^{2} \subset \cdots \subset \mathcal{F}^{r-1} \subset \mathcal{F}^{r}=$ $\mathcal{L}(U)$,

$$
\mathcal{F}^{s} \stackrel{\text { dfn }}{=}\left\{\lambda \in \mathcal{L}(U) \mid 0=\lambda^{1}, \ldots, \lambda^{r-s}\right\}, \quad 0 \leq s \leq r
$$

and (ii) a decomposition $\mathcal{L}(U)=\mathcal{L}^{0} \sqcup \mathcal{L}^{1} \sqcup \cdots \sqcup \mathcal{L}^{r}$,

$$
\mathcal{L}^{s} \stackrel{\text { dfn }}{=} \mathcal{F}^{s} \backslash \mathcal{F}^{s-1} \stackrel{(4.6)}{=}\left\{\lambda \in \mathcal{L}(U) \mid \lambda^{r-s}=0, \lambda^{r-s+1}=1\right\}, \quad 0 \leq s \leq r,
$$

with the convention that $\mathcal{F}^{-1}=\emptyset$.

The spin representation is of dimension $2^{r}$. By (2.8) and (2.20),

$$
\frac{1}{2}\left(2^{r}-1\right)=m=\omega_{r}\left(\mathrm{~T}_{\varphi}\right) .
$$


The spin representation: examples. Before continuing with the proof, we consider some examples.

Example 4.9. If $r=2$, then $\operatorname{dim}_{\mathbb{C}} U=4$, and the weights $\lambda \in \mathcal{L}$ are

$$
\text { (00), (01), (11), (12). }
$$

This forces $\mathrm{T}_{\varphi}=\mathrm{T}^{1}+\mathrm{T}^{2}$. The $\mathrm{T}_{\varphi}$-graded decomposition of $U$ is $U_{3 / 2} \oplus U_{1 / 2} \oplus U_{-1 / 2} \oplus U_{-3 / 2}$. Thus (2.8) and (2.9) hold. Moreover, $\mathrm{T}^{\mathrm{cpt}}=0$, so that $\omega_{r}\left(\mathrm{~T}^{\mathrm{cpt}}\right)$ is even; thus, $U$ is real.

Example 4.10. If $r=3$, then $\operatorname{dim}_{\mathbb{C}} U=8$, and the weights are

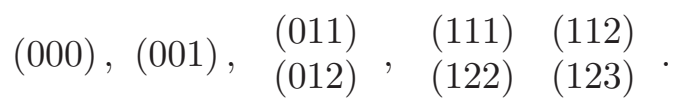

This forces $\mathrm{T}_{\varphi}=2 \mathrm{~T}^{1}+\mathrm{T}^{2}+\mathrm{T}^{3}$. For this grading element, $\omega_{r}\left(\mathrm{~T}_{\varphi}\right)=\frac{1}{2} 7$, as required by (4.8), and the eigenvalues $\left\{ \pm \frac{1}{2} p \mid p=1,3,5,7\right\}$ of $\mathrm{T}_{\varphi}$ are all multiplicity free. Moreover, $\mathrm{T}^{\mathrm{cpt}}=2 \mathrm{~T}^{1}$, so that $\omega_{r}\left(\mathrm{~T}^{\mathrm{cpt}}\right)=1$ is odd; thus, $U$ is quaternionic.

Example 4.11. If $r=4$, then $\operatorname{dim}_{\mathbb{C}} U=16$, and the weights are

$$
\begin{array}{lllll} 
& & (0111) & (1111) & (1112) \\
(0000),(0001), & (0011) & (0112) & (1122) & (1222) \\
(0012) & (0122) \\
& (0123) & (1123) & (1223) \\
\end{array}
$$

So, in order to have multiplicity-free, $\mathrm{T}_{\varphi}$-eigenvalues, we must have $n_{4}=n_{3}=1, n_{2}=2$ and $n_{1}=4$. This choice does indeed give consecutive eigenvalues $\left\{ \pm \frac{1}{2} p \mid p=1,3,5, \ldots, 15\right\}$, and in particular satisfies $\omega_{4}\left(\mathrm{~T}_{\varphi}\right)=\frac{1}{2}(4+2 \cdot 2+3 \cdot 1+4 \cdot 1)=\frac{1}{2} 15$, as required by $(4.8)$.

\begin{tabular}{|c|c|c|c|c|c|c|c|}
\hline (00000) & (001 & & & & & & \\
\hline & & & & & & & \\
\hline (00011) & $(00122)$ & & & & & & \\
\hline (00012) & (00123) & (01233) & (01234) & (12234) & (12334) & (12344) & (12345) \\
\hline
\end{tabular}
Moreover, $\mathrm{T}^{\mathrm{cpt}}=2\left(\mathrm{~T}^{1}+\mathrm{T}^{2}\right)$, so that $\omega_{r}\left(\mathrm{~T}^{\mathrm{cpt}}\right)=1+2=3$ is odd; thus, $U$ is quaternionic.

Example 4.12. If $r=5$, then $\operatorname{dim}_{\mathbb{C}} U=32$, and the weights are

In order for $\mathrm{T}_{\varphi}$ to have the consecutive, multiplicity-free eigenvalues required by (2.9), we must have $\mathrm{T}_{\varphi}=8 \mathrm{~T}^{1}+4 \mathrm{~T}^{2}+2 \mathrm{~T}^{3}+\mathrm{T}^{4}+\mathrm{T}^{5}$. For this grading element, (2.8) holds. Moreover, $\mathrm{T}^{\mathrm{cpt}}=2\left(\mathrm{~T}^{1}+\mathrm{T}^{2}+\mathrm{T}^{3}\right)$, so that $\omega_{r}\left(\mathrm{~T}^{\mathrm{cpt}}\right)=1+2+3$ is even; thus, $U$ is real.

The spin representation: completing the proof. We now return to the proof of Theorem 4.1. Observe that

$$
\mathcal{F}^{2}=\{(0 \cdots 0),(0 \cdots 01),(0 \cdots 011),(0 \cdots 012)\} .
$$

The $\mathrm{T}_{\varphi}$-eigenvalues for these weights are

$$
\mathcal{F}^{2}\left(\mathrm{~T}_{\varphi}\right)=\left\{m, m-n_{r}, m-\left(n_{r-1}+n_{r}\right), m-\left(n_{r-1}+2 n_{r}\right)\right\} .
$$

By (4.6), all other weights of the representation satisfy

$$
\lambda\left(\mathrm{T}_{\varphi}\right) \leq m-\left(n_{r-2}+n_{r-1}+n_{r}\right) \text { for all } \lambda \notin \mathcal{F}^{2} .
$$


Therefore, the requirement (2.9) forces

$$
n_{r}=n_{r-1}=1
$$

These first four eigenvalues are

$$
\mathcal{F}^{2}\left(\mathrm{~T}_{\varphi}\right)=\{m-p \mid p=0,1,2,3\} .
$$

As noted in (4.13), the largest $\mathrm{T}_{\varphi}$-eigenvalue $\lambda\left(\mathrm{T}_{\varphi}\right)$ among the $\lambda \notin \mathcal{F}^{2}$ is $m-\left(n_{r-2}+\right.$ $\left.n_{r-1}+n_{r}\right)=m-2-n_{r-2}$, and is realized by the weight $\lambda=(0 \cdots 0111)$. Given (4.14), the requirement (2.9) then forces

$$
n_{r-2}=2 \text {. }
$$

Note that $\mathcal{L}^{3}=\{(0 \cdots 0111),(0 \cdots 0112),(0 \cdots 0122),(0 \cdots 0123)\}$, and the eigenvalues

$$
\mathcal{L}^{3}\left(\mathrm{~T}_{\varphi}\right)=\{m-p \mid p=4,5,6,7\} \quad \text { and } \quad \mathcal{F}^{3}\left(\mathrm{~T}_{\varphi}\right)=\{m-p \mid p=0,1, \ldots, 7\} .
$$

We will establish (4.2) by induction. We will need the formula

$$
2^{t+1}=1+1+2+4+8+\cdots+2^{t-1}+2^{t},
$$

which is easily confirmed by an inductive argument. Suppose that there exists $2 \leq \mathrm{s} \leq r-2$ such that

$$
\begin{aligned}
n_{r-t} & =2^{t-1} \text { and } \\
\mathcal{L}^{t+1}\left(\mathrm{~T}_{\varphi}\right) & =\left\{m-p \mid p=2^{t}, 2^{t}+1, \ldots, 2^{t+1}-1\right\},
\end{aligned}
$$

for all $2 \leq t \leq \mathrm{s}$. In the preceding paragraph, we saw that (4.16) holds for $\mathrm{s}=2$. To complete the proof of the lemma we need to show that

$$
\begin{aligned}
n_{r-\mathrm{s}-1} & =2^{\mathrm{s}} \text { and } \\
\mathcal{L}^{\mathrm{s}+2}\left(\mathrm{~T}_{\varphi}\right) & =\left\{m-p \mid p=2^{\mathrm{s}+1}, 2^{\mathrm{s}+1}+1, \ldots, 2^{\mathrm{s}+2}-1\right\} .
\end{aligned}
$$

Note that (4.16b) implies that the eigenvalues

$$
\mathcal{F}^{\mathrm{s}+1}\left(\mathrm{~T}_{\varphi}\right)=\left\{m-p \mid p=0,1,2, \ldots, 2^{\mathrm{s}+1}-1\right\} .
$$

By (4.6) and (4.16b),

$$
m-\left(n_{r-s-1}+n_{r-s}+\cdots+n_{r}\right)=\max \left\{\lambda\left(\mathrm{T}_{\varphi}\right) \mid \lambda \notin \mathcal{F}^{s+1}\right\}
$$

is realized by the weight $\lambda=\left(0^{r-s-2} 1^{s+2}\right)$. By (4.15), (4.16a) an (4.19) the largest eigenvalue amongst the $\left\{\lambda\left(\mathrm{T}_{\varphi}\right) \mid \lambda \notin \mathcal{F}^{\mathrm{s}+1}\right\}$ is $m-\left(n_{r-\mathrm{s}-1}+2^{\mathrm{s}-1}+2^{\mathrm{s}-2}+\cdots+2+1+1\right)=$ $m-\left(n_{r-s-1}+2^{\mathrm{s}}\right)$. Given (4.18) and (2.9), this observation, forces $n_{r-s-1}+2^{\mathrm{s}}=2^{\mathrm{s}+1}$. That is, $n_{r-\mathrm{s}-1}=2^{\mathrm{s}}$. This establishes (4.17a).

It remains to prove (4.17b). Given $\lambda \in \mathcal{L}^{\mathrm{s}+2}$, note that (4.6) and (4.7b) imply either $\lambda^{r-\mathrm{s}}=1$ or $\lambda^{r-\mathrm{s}}=2$. In particular, we have a disjoint union

$$
\mathcal{L}^{\mathrm{s}+2}=\mathcal{L}_{1}^{\mathrm{s}+2} \sqcup \mathcal{L}_{2}^{\mathrm{s}+2},
$$

given by

$$
\mathcal{L}_{i}^{\mathrm{s}+2} \stackrel{\text { dfn }}{=}\left\{\lambda \in \mathcal{L}^{\mathrm{s}+2} \mid \lambda^{r-\mathrm{s}}=i\right\}
$$


Elements of $\mathcal{L}_{1}^{\mathbf{s}+2}$ are of the form $\lambda=\left(0 \cdots 011 \lambda^{r-\mathrm{s}+1} \cdots \lambda^{r}\right)$. The map $\lambda \mapsto \lambda+\sigma_{r-\mathrm{s}-1}=$ $\left(0 \cdots 001 \lambda^{r-\mathrm{s}-1} \cdots \lambda^{r}\right)$ defines a bijection $\mathcal{L}_{1}^{\mathrm{s}+2} \rightarrow \mathcal{L}^{\mathrm{s}+1}$. Given $(4.16 \mathrm{~b})$, we have

$$
\begin{aligned}
\mathcal{L}_{1}^{\mathrm{s}+2}\left(\mathrm{~T}_{\varphi}\right) & =\left\{\lambda\left(\mathrm{T}_{\varphi}\right)-2^{\mathrm{s}} \mid \lambda \in \mathcal{L}^{\mathrm{s}+1}\right\} \\
& =\left\{m-p \mid p=2^{\mathrm{s}+1}, 2^{\mathrm{s}+1}+1, \ldots, 3 \cdot 2^{\mathrm{s}}-1\right\} .
\end{aligned}
$$

Likewise, elements of $\mathcal{L}_{2}^{\mathrm{s}+2}$ are of the form $\lambda=\left(0 \cdots 012 \lambda^{r-\mathrm{s}+1} \cdots \lambda^{r}\right)$, and the map $\lambda \mapsto$ $\lambda-\left(\sigma_{r-s-1}+\sigma_{r-s}+\cdots+\sigma_{r}\right)=\left(0 \cdots 001\left(\lambda^{r-s+1}-1\right) \cdots\left(\lambda^{r}-1\right)\right)$ defines a bijection $\mathcal{L}_{2}^{\mathrm{s}+2} \rightarrow \mathcal{L}^{\mathrm{s}+1}$.

With (4.15) and (4.16b), this implies that the eigenvalues

$$
\begin{aligned}
\mathcal{L}_{2}^{\mathrm{s}+2}\left(\mathrm{~T}_{\varphi}\right) & =\left\{\lambda\left(\mathrm{T}_{\varphi}\right)-\left(2^{\mathrm{s}}+2^{\mathrm{s}-1}+\cdots+2+1+1\right) \mid \lambda \in \mathcal{L}^{\mathrm{s}+1}\right\} \\
& =\left\{m-p \mid p=3 \cdot 2^{\mathrm{s}}, 3 \cdot 2^{\mathrm{s}}+1, \ldots, 2^{\mathrm{s}+2}-1\right\} .
\end{aligned}
$$

Equations (4.20) establish (4.17b). This completes the proof of (4.2).

By $(2.3), \mathrm{T}^{\mathrm{cpt}}=2\left(\mathrm{~T}^{1}+\cdots+\mathrm{T}^{r-2}\right)$. Thus, $\mu\left(\mathrm{T}^{\mathrm{cpt}}\right)=\omega_{r}\left(\mathrm{~T}^{\mathrm{cpt}}\right)=\sum_{1}^{r-2} i=\frac{1}{2}(r-2)(r-1)$. By (2.4), the self-dual $U$ is real, as required by (2.11), if and only if $(r-2)(r-1) \in 4 \mathbb{Z}$.

Proof of Theorem 4.3. Let $U$ be the irreducible $\mathfrak{s o}_{2 r} \mathbb{C}$-module associated to $V_{\mathbb{R}}$, cf. $\S 2.4$. If $(V, \varphi)$ is principal, $U$ is weight multiplicity-free by (2.13). By Theorem 2.14(d), $U$ is either the standard representation $\mathbb{C}^{2 r}$ of highest weight

$$
\omega_{1}=\sigma_{1}+\cdots+\sigma_{r-2}+\frac{1}{2}\left(\sigma_{r-1}+\sigma_{r}\right),
$$

or one of the spin representations with highest weight

$$
\begin{aligned}
\omega_{r-1} & =\frac{1}{2}\left(\sigma_{1}+2 \sigma_{2}+\cdots+(r-2) \sigma_{r-2}\right)+\frac{1}{4}\left(r \sigma_{r-1}+(r-2) \sigma_{r}\right), \\
\omega_{r} & =\frac{1}{2}\left(\sigma_{1}+2 \sigma_{2}+\cdots+(r-2) \sigma_{r-2}\right)+\frac{1}{4}\left((r-2) \sigma_{r-1}+r \sigma_{r}\right) .
\end{aligned}
$$

The standard representation. Suppose that $U$ is the standard representation. Then $U$ is self-dual, and so either real or quaternionic. By $(2.11)$, if $(V, \varphi)$ is principal, then $U$ is real, so that $V_{\mathbb{C}}=U$. Therefore, the weights of $V_{\mathbb{C}}$ are

$$
\begin{aligned}
\Lambda\left(V_{\mathbb{C}}\right)= & \left\{\omega_{1}, \omega_{1}-\left(\sigma_{1}+\cdots+\sigma_{r-2}+\sigma_{r}\right)\right\} \cup\left\{\omega_{1}-\left(\sigma_{1}+\cdots+\sigma_{i}\right) \mid 1 \leq i \leq r\right\} \\
& \cup\left\{\omega_{1}-\left(\sigma_{1}+\cdots+\sigma_{i-1}\right)-2\left(\sigma_{i}+\cdots+\sigma_{r-2}\right)-\sigma_{r-1}-\sigma_{r} \mid 1 \leq i \leq r-2\right\} .
\end{aligned}
$$

It is straight-forward to confirm that

$$
\begin{aligned}
& \mathrm{T}_{\varphi}=\mathrm{T}^{1}+\cdots+\mathrm{T}^{r-2}+\mathrm{T}^{r-1}+2 \mathrm{~T}^{r} \\
& \mathrm{~T}_{\varphi}=\mathrm{T}^{1}+\cdots+\mathrm{T}^{r-2}+2 \mathrm{~T}^{r-1}+\mathrm{T}^{r}
\end{aligned} \text { and }
$$

are the only grading elements satisfying (2.8) and yielding (multiplicity-free) eigenvalues (2.9). For these two grading elements we have $\mathrm{T}^{\mathrm{cpt}}=2 \mathrm{~T}^{r-1}$ and $\mathrm{T}^{\mathrm{cpt}}=2 \mathrm{~T}^{r}$, respectively. Therefore, $\omega_{1}\left(\mathrm{~T}^{\mathrm{cpt}}\right)=1$ and $U$ is quaternionic, by (2.4), contradicting (2.11). It follows that there exists no principle Hodge representation $(V, \varphi)$ of $G$ such that $U=\mathbb{C}^{2 r}$.

Remark 4.22. The argument above yields the following: Let $G$ be a Hodge group with complex Lie algebra $\mathfrak{g}_{\mathbb{C}}=\mathfrak{s o}_{2 r} \mathbb{C}$. Assume that $(V, \varphi)$ is a Hodge representation with the property that $V_{\mathbb{R}}$ is an irreducible $G_{\mathbb{R}}$-module, and the associated $\mathfrak{g}_{\mathbb{C}}$-module $U$ (§2.4) is the standard representation. Let $\mathrm{T}_{\varphi}$ be the associated grading element (\$2.3), and assume the 
normalization (2.2) holds. Then Hodge numbers are $\mathbf{h}=(2,2, \ldots, 2)$ if and only if one of (4.21) holds.

The spin representation, $r$ even. If $r$ is even, then the spin representations are self-dual. Arguing as above in the case of the standard representation, it is necessarily the case that $r>4$. (Else $U$ is quaternionic.) The two spin representations have highest weight $\mu=\omega_{r-1}$ and $\mu=\omega_{r}$. The arguments for the two cases are symmetric, so we will assume $\mu=\omega_{r}$. The weights of $U$ are parameterized by

$$
\begin{aligned}
\mathcal{L}(U)=\left\{\lambda=\left(\lambda^{1}, \ldots, \lambda^{r}\right) \in \mathbb{Z}^{r} \quad \mid\right. & \lambda^{1}, \lambda^{r}+\lambda^{r-1}-\lambda^{r-2}, \lambda^{r}-\lambda^{r-1} \in\{0,1\}, \\
& \left.\lambda^{i}-\lambda^{i-1} \in\{0,1\}, \forall 1<i \leq r-2\right\}
\end{aligned}
$$

specifically,

$$
\Lambda(U)=\left\{\omega_{r}-\lambda^{i} \sigma_{i} \mid \lambda \in \mathcal{L}(U)\right\} .
$$

Set $m=\omega_{r}\left(\mathrm{~T}_{\varphi}\right)$. Given a weight $\lambda \in \mathcal{L}(U)$, the corresponding $\mathrm{T}_{\varphi}$-eigenvalue is $\lambda\left(\mathrm{T}_{\varphi}\right)=$ $m-\sum_{i} \lambda^{i} n_{i}$. As in the proof of Theorem 4.1(b), we define a filtration $\mathcal{F}^{3} \subset \mathcal{F}^{4} \subset \cdots \subset$ $\mathcal{F}^{r-1} \subset \mathcal{F}^{r}=\mathcal{L}(U)$

$$
\mathcal{F}^{s} \stackrel{\text { dfn }}{=}\left\{\lambda \in \mathcal{L}(U) \mid 0=\lambda^{1}, \ldots, \lambda^{r-s}\right\}
$$

and decomposition $\mathcal{L}(U)=\mathcal{L}^{3} \sqcup \mathcal{L}^{4} \sqcup \cdots \sqcup \mathcal{L}^{r}$

$$
\mathcal{L}^{s} \stackrel{\text { dfn }}{=} \mathcal{F}^{s} \backslash \mathcal{F}^{s-1}=\left\{\lambda \in \mathcal{L}(U) \mid \lambda^{r-s}=0, \lambda^{r-s+1}=1\right\},
$$

for $4 \leq s \leq r$, and

$$
\mathcal{L}^{3} \stackrel{\text { dfn }}{=} \mathcal{F}^{3}=\{(0 \cdots 0),(0 \cdots 01),(0 \cdots 0101),(0 \cdots 0111)\} .
$$

The eigenvalues of $\mathcal{L}^{3}$ are

$$
\mathcal{L}^{3}\left(\mathrm{~T}_{\varphi}\right)=\left\{m, m-n_{r}, m-\left(n_{r-2}+n_{r}\right), m-\left(n_{r-2}+n_{r-1}+n_{r}\right)\right\} .
$$

In general,

$$
\max \left\{\lambda\left(\mathrm{T}_{\varphi}\right) \mid \lambda \notin \mathcal{F}^{s}\right\}=m-\left(n_{r-s}+n_{r-s+1}+\cdots+n_{r-2}+n_{r}\right)
$$

is realized by the weight $\lambda=\left(0^{r-s-1} 1^{s-1} 01\right)$. Therefore, $\max \left\{\lambda\left(\mathrm{T}_{\varphi}\right) \mid \lambda \notin \mathcal{F}^{3}=\mathcal{L}^{3}\right\}=$ $m-\left(n_{r-3}+n_{r-2}+n_{r}\right)$. So, given (4.25), the condition (2.9) forces

$$
n_{r}=n_{r-2}=1 \text {. }
$$

This updates (4.25) to $\mathcal{L}^{3}\left(\mathrm{~T}_{\varphi}\right)=\left\{m, m-1, m-2, m-\left(2+n_{r-1}\right)\right\}$. From $\mathcal{L}^{4}=$ $\{(0 \cdots 01101),(0 \cdots 01111),(0 \cdots 01211),(0 \cdots 01212)\}$, we see that the eigenvalues of $\mathcal{F}^{4}=\mathcal{L}^{3} \sqcup \mathcal{L}^{4}$ are

$$
\begin{aligned}
\mathcal{F}^{4}\left(\mathrm{~T}_{\varphi}\right)=\{ & m, m-1, m-2, m-\left(2+n_{r-1}\right), m-\left(2+n_{r-3}\right), \\
& m-\left(2+n_{r-3}+n_{r-1}\right), m-\left(3+n_{r-3}+n_{r-1}\right), \\
& \left.m-\left(4+n_{r-3}+n_{r-1}\right)\right\} .
\end{aligned}
$$

Given (4.26) and (4.27), the condition (2.9) implies one of the following two holds

$$
\begin{aligned}
& n_{r-1}=1 \text { and } n_{r-3}=2 \text {, or } \\
& n_{r-1}=2 \text { and } n_{r-3}=1 \text {. }
\end{aligned}
$$


I claim that (4.28b) is not possible. For, if $(4.28 \mathrm{~b})$ were to hold, then both weights $(0 \cdots 011212),(0 \cdots 012211) \in \mathcal{L}^{5}$ would have eigenvalue $m-\left(n_{r-4}+7\right)$, contradicting the requirement (2.7) that the eigenvalues have multiplicity one. Therefore (4.28a) holds. The eigenvalues of $\mathcal{F}^{4}$ are

$$
\mathcal{F}^{4}\left(\mathrm{~T}_{\varphi}\right)=\{m-p \mid p=0,1, \ldots, 7\} .
$$

We will establish (4.4) by induction. Suppose that there exists $2 \leq \mathrm{s} \leq r-2$ such that

$$
\begin{aligned}
n_{r-s} & =2^{s-2} \text { and } \\
\mathcal{F}^{s+1}\left(\mathrm{~T}_{\varphi}\right) & =\left\{m-p \mid p=0,1, \ldots, 2^{s}-1\right\},
\end{aligned}
$$

for all $2 \leq s \leq \mathrm{s}$. The discussion above establishes these inductive hypotheses for $\mathrm{s}=3$. To complete the proof of (4.4) we need to show that

$$
\begin{aligned}
n_{r-\mathrm{s}-1} & =2^{\mathrm{s}-1} \text { and } \\
\mathcal{L}^{\mathrm{s}+2} & =\left\{m-p \mid p=2^{\mathrm{s}}, 2^{\mathrm{s}}+1, \ldots, 2^{\mathrm{s}+1}-1\right\} .
\end{aligned}
$$

Given (4.29b), the requirement (2.9) that the $\mathrm{T}_{\varphi}$-eigenvalues be consecutive forces the largest eigenvalue $\lambda\left(\mathrm{T}_{\varphi}\right)$ among the $\lambda \notin \mathcal{F}^{s+1}$ to be $m-2^{\mathrm{s}}$. By (4.26), (4.29a) and (4.15), this largest eigenvalue is $m-n_{r-\mathrm{s}-1}+2^{\mathrm{s}-2}+2^{\mathrm{s}-3}+\cdots+2+1+1=m-n_{r-\mathrm{s}-1}+2^{\mathrm{s}-1}$. Therefore, $n_{r-s-1}=2^{s-1}$, establishing (4.30a).

To prove $(4.30 \mathrm{~b})$, note that (4.23a) implies that

$$
\mathcal{L}^{\mathrm{s}+2}=\mathcal{L}_{1}^{\mathrm{s}+2} \sqcup \mathcal{L}_{2}^{\mathrm{s}+2}
$$

where

$$
\mathcal{L}_{i}^{\mathrm{s}+2}=\left\{\lambda \in \mathcal{L}(U) \mid \lambda^{r-\mathrm{s}-2}=0, \lambda^{r-\mathrm{s}-1}=1, \lambda^{r-\mathrm{s}}=i\right\} .
$$

The map $\lambda=(0 \cdots 011 \cdots) \mapsto \lambda+\sigma_{r-s-1}=(0 \cdots 001 \cdots)$ defines a bijection $\mathcal{L}_{1}^{\mathbf{s}+2} \rightarrow \mathcal{L}^{\mathbf{s}+1}$. By (4.29b), the eigenvalues of $\mathcal{L}^{\mathrm{s}+1}=\mathcal{F}^{\mathrm{s}+1} \backslash \mathcal{F}^{\mathrm{s}}$ are $\mathcal{L}^{\mathrm{s}+1}\left(\mathrm{~T}_{\varphi}\right)=\left\{m-p \mid p=2^{\mathrm{s}-1}, 2^{\mathrm{s}-1}+\right.$ $\left.1, \ldots, 2^{\mathrm{s}}-1\right\}$. So, by $(4.29 \mathrm{~b})$, the eigenvalues of $\mathcal{L}_{1}^{\mathrm{s}+2}$ are

$$
\mathcal{L}_{1}^{\mathrm{s}+2}\left(\mathrm{~T}_{\varphi}\right)=\left\{\lambda\left(\mathrm{T}_{\varphi}\right)-n_{r-\mathrm{s}-1} \mid \lambda \in \mathcal{L}^{\mathrm{s}+1}\right\}=\left\{m-p \mid p=2^{\mathrm{s}}, 2^{\mathrm{s}}+1, \ldots, 3 \cdot 2^{\mathrm{s}-1}-1\right\}
$$

Similarly, the map

$$
\lambda \mapsto \begin{cases}\lambda+\sigma_{r-\mathrm{s}-1}+\cdots+\sigma_{r-2}+\sigma_{r}, & \text { if } \lambda^{r}=\lambda^{r-1}+1 \\ \lambda+\sigma_{r-\mathrm{s}-1}+\cdots+\sigma_{r-1}, & \text { if } \lambda^{r}=\lambda^{r-1}\end{cases}
$$

defines a bijection $\mathcal{L}_{2}^{\mathrm{s}+2} \rightarrow \mathcal{L}^{\mathrm{s}+1}$. Arguing as above, and making use of (4.15), the eigenvalues of $\mathcal{L}_{2}^{\mathrm{s}+2}$ are

$$
\begin{aligned}
\mathcal{L}_{2}^{\mathrm{s}+2}\left(\mathrm{~T}_{\varphi}\right) & =\left\{\lambda\left(\mathrm{T}_{\varphi}\right)-\left(2^{\mathrm{s}-1}+2^{\mathrm{s}-2}+\cdots+2+1+1\right) \mid \lambda \in \mathcal{L}^{\mathrm{s}+1}\right\} \\
& =\left\{\lambda\left(\mathrm{T}_{\varphi}\right)-2^{\mathrm{s}} \mid \lambda \in \mathcal{L}^{\mathrm{s}+1}\right\}=\left\{m-p \mid p=3 \cdot 2^{\mathrm{s}-1}, \cdots, 2^{\mathrm{s}+1}-1\right\} .
\end{aligned}
$$

Item (4.30b) now follows from (4.31), and (4.4) is established.

By $(2.3), \mathrm{T}^{\mathrm{cpt}}=2\left(\mathrm{~T}^{1}+\cdots+\mathrm{T}^{r-3}\right)$. Thus, $\mu\left(\mathrm{T}^{\mathrm{cpt}}\right)=\omega_{r}\left(\mathrm{~T}^{\mathrm{cpt}}\right)=\sum_{1}^{r-3} i=\frac{1}{2}(r-3)(r-2)$. By (2.4), the self-dual $U$ is real, as required by $(2.11)$, if and only if $(r-3)(r-2) \in 4 \mathbb{Z}$. 
The spin representation, $r$ odd. If $r$ is odd, then $U_{\omega_{r-1}}^{*}=U_{\omega_{r}}$. In particular, the spin representations are not self-dual, and $V_{\mathbb{C}}=U_{\omega_{r-1}} \oplus U_{\omega_{r}}$. Without loss of generality, we will assume that the representation $U$ associated to $V_{\mathbb{R}}$ in $\S 2.4$ is $U_{\omega_{r}}$; that is, $\mu=\omega_{r}$. (With the normalization (2.21), this will yield the first grading element of (4.5). Taking $\mu=\omega_{r-1}$ yields the second grading element. The two arguments are symmetric, and we will give only the first.)

The weights of $U$ are given by (4.23). The weights of $U^{*}$ are parameterized by

$$
\begin{aligned}
& \mathcal{L}\left(U^{*}\right)=\left\{\mu=\left[\mu_{1}, \ldots, \mu_{r}\right] \in \mathbb{Z}^{r} \quad \mid \quad \mu^{1}, \mu^{r}+\mu^{r-1}-\mu^{r-2}, \mu^{r-1}-\mu^{r} \in\{0,1\},\right. \\
&\left.\mu^{i}-\mu^{i-1} \in\{0,1\}, \forall 1<i \leq r-2\right\}
\end{aligned}
$$

specifically,

$$
\Lambda\left(U^{*}\right)=\left\{\omega_{r-1}-\mu^{i} \sigma_{i} \mid \mu \in \mathcal{L}\left(U^{*}\right)\right\} .
$$

Following (4.24), we define a filtration $\mathcal{G}^{3} \subset \mathcal{G}^{4} \subset \cdots \subset \mathcal{G}^{r-1} \subset \mathcal{G}^{r}=\mathcal{L}\left(U^{*}\right)$

$$
\mathcal{G}^{s} \stackrel{\text { dfn }}{=}\left\{\mu \in \mathcal{L}\left(U^{*}\right) \mid 0=\mu^{1}, \ldots, \mu^{r-s}\right\}
$$

and decomposition $\mathcal{L}\left(U^{*}\right)=\mathcal{M}^{3} \sqcup \mathcal{M}^{4} \sqcup \cdots \sqcup \mathcal{M}^{r}$

$$
\mathcal{M}^{s} \stackrel{\text { dfn }}{=} \mathcal{G}^{s} \backslash \mathcal{G}^{s-1}=\left\{\mu \in \mathcal{L}\left(U^{*}\right) \mid \mu^{r-s}=0, \mu^{r-s+1}=1\right\},
$$

for $4 \leq s \leq r$, and

$$
\mathcal{M}^{3} \stackrel{\text { dfn }}{=} \mathcal{G}^{3}=\{[0 \cdots 0],[0 \cdots 010],[0 \cdots 0110],[0 \cdots 0111]\} .
$$

I claim that

$$
n_{r}=3 \text { and } n_{r-1}=1 .
$$

To see this, recall that $m=\omega_{r}\left(\mathrm{~T}_{\varphi}\right)$, by $(2.20)$, and $m^{*}=\omega_{r-1}\left(\mathrm{~T}_{\varphi}\right)$, by (2.21). By Lemma $2.22(\mathrm{~b})$,

$$
0<m-m^{*}=\omega_{r}\left(\mathrm{~T}_{\varphi}\right)-\omega_{r-1}\left(\mathrm{~T}_{\varphi}\right)=\frac{1}{2}\left(n_{r}-n_{r-1}\right) \in \mathbb{Z} .
$$

With (2.17), this implies $0<n_{r}-n_{r-1} \in 2 \mathbb{Z}$. Therefore, $n_{r} \geq 3$. By Lemma 2.22(b),

$$
m-m^{*}=1
$$

and $n_{r-1}=1$. Thus, $n_{r}=3$.

The eigenvalues associated to the weights $\mathcal{L}^{3} \cup \mathcal{M}^{3}$ are

$$
\begin{aligned}
\mathcal{L}^{3}\left(\mathrm{~T}_{\varphi}\right) & =\left\{m, m-3, m-3-n_{r-2}, m-4-n_{r-2}\right\} \\
\mathcal{M}^{3}\left(\mathrm{~T}_{\varphi}\right) & =\left\{m-1, m-2, m-2-n_{r-2}, m-5-n_{r-2}\right\} .
\end{aligned}
$$

If $n_{r-2}=1$, then the eigenvalue $m-3$ will have multiplicity greater than one; so, the condition (2.10) that the $\mathrm{T}_{\varphi}$-eigenvalues have multiplicity one forces

$$
n_{r-2}>1 .
$$

In analogy with (4.26),

$$
\begin{aligned}
\max \left\{\mu\left(\mathrm{T}_{\varphi}\right) \mid \mu \notin \mathcal{G}^{s}\right\} & =\omega_{r-1}\left(\mathrm{~T}_{\varphi}\right)-\left(n_{r-s}+n_{r-s+1}+\cdots+n_{r-1}\right) \\
& =m-1-\left(n_{r-s}+n_{r-s+1}+\cdots+n_{r-1}\right)
\end{aligned}
$$


is realized by $\lambda=\left(0^{r-s-1} 1^{s} 0\right)$. By (4.26) and (4.36) the largest $\mathrm{T}_{\varphi}$-eigenvalues amongst the $\lambda \notin \mathcal{L}^{3}=\mathcal{F}^{3}$ and the $\mu \notin \mathcal{M}^{3}=\mathcal{G}^{3}$ are

$$
m-\left(n_{r-3}+n_{r-2}+3\right) \text { and } m-\left(n_{r-3}+n_{r-2}+2\right) \text {. }
$$

So, if $n_{r-2}>2$, then (2.17), (4.35) and (4.37) imply $m-4$ will not appear as an eigenvalue. This contradicts the requirement (2.9) that the $\mathrm{T}_{\varphi}$-eigenvalues be consecutive; thus

$$
n_{r-2}=2 \text {. }
$$

From (4.34), (4.35) and (4.38), we see that the $\mathrm{T}_{\varphi}$-eigenvalues of $\mathcal{L}^{3} \cup \mathcal{M}^{3}$ are

$$
\mathcal{L}^{3}\left(\mathrm{~T}_{\varphi}\right) \cup \mathcal{M}^{3}\left(\mathrm{~T}_{\varphi}\right)=\{m-p \mid p=0,1, \ldots, 7\} .
$$

We will complete the proof that $\mathrm{T}_{\varphi}$ is the first grading element of (4.5) by induction. Suppose that there exists $2 \leq \mathrm{s} \leq r-2$ such that:

$$
\begin{aligned}
n_{r-s} & =2^{s-1} \text { and } \\
\mathcal{F}^{s+1}\left(\mathrm{~T}_{\varphi}\right) \cup \mathcal{G}^{s+1}\left(\mathrm{~T}_{\varphi}\right) & =\left\{m-p \mid p=0,1, \ldots, 2^{s+1}-1\right\},
\end{aligned}
$$

for all $2 \leq s \leq \mathrm{s}$. Keeping in mind that $\mathcal{L}^{3}=\mathcal{F}^{3}$ and $\mathcal{M}^{3}=\mathcal{G}^{3}$, we have seen that this inductive hypothesis holds for $\mathrm{s}=2$. To complete the induction (and proof of the lemma), we must show that

$$
\begin{aligned}
n_{r-\mathrm{s}-1} & =2^{\mathrm{s}} \text { and } \\
\mathcal{L}^{\mathrm{s}+2}\left(\mathrm{~T}_{\varphi}\right) \cup \mathcal{M}^{\mathrm{s}+2}\left(\mathrm{~T}_{\varphi}\right) & =\left\{m-p \mid p=2^{\mathrm{s}+1}, 2^{\mathrm{s}+1}+1, \ldots, 2^{\mathrm{s}+2}-1\right\} .
\end{aligned}
$$

By (4.26), (4.39a) and (4.15),

$$
\begin{aligned}
\max \left\{\lambda\left(\mathrm{T}_{\varphi}\right) \mid \lambda \notin \mathcal{F}^{\mathrm{s}+1}\right\} & =m-\left(n_{r-\mathrm{s}-1}+2^{\mathrm{s}-1}+\cdots+4+2+3\right) \\
& =m-\left(n_{r-\mathrm{s}-1}+2^{\mathrm{s}}+1\right) .
\end{aligned}
$$

Similarly, (4.36), (4.39a) and (4.15) yield

$$
\begin{aligned}
\max \left\{\mu\left(\mathrm{T}_{\varphi}\right) \mid \mu \notin \mathcal{G}^{\mathrm{s}+1}\right\} & =m-1-\left(n_{r-\mathrm{s}-1}+2^{\mathrm{s}-1}+\cdots+4+2+1\right) \\
& =m-\left(n_{r-\mathrm{s}-1}+2^{\mathrm{s}}\right) .
\end{aligned}
$$

On the other hand $(4.39 \mathrm{~b})$ and the requirement $(2.9)$ that the eigenvalues of $V_{\mathbb{C}}$ be consecutive imply $\max \left\{\lambda\left(\mathrm{T}_{\varphi}\right), \mu\left(\mathrm{T}_{\varphi}\right) \mid \lambda \notin \mathcal{F}^{\mathrm{s}+1}, \mu \notin \mathcal{G}^{\mathrm{s}+1}\right\}=m-2^{\mathrm{s}+1}$. Therefore, $n_{r-\mathrm{s}-1}=2^{\mathrm{s}}$, establishing (4.40a).

In analogy with (4.31a), observe that (4.32a) implies

$$
\mathcal{M}^{\mathrm{s}+2}=\mathcal{M}_{1}^{\mathrm{s}+2} \cup \mathcal{M}_{2}^{\mathrm{s}+2},
$$

where

$$
\mathcal{M}_{i}^{\mathrm{s}+2}=\left\{\mu \in \mathcal{L}\left(U^{*}\right) \mid \mu^{r-\mathrm{s}-2}=0, \mu^{r-\mathrm{s}-1}=1, \mu^{r-\mathrm{s}}=i\right\} .
$$

The assignment $\nu \mapsto \nu+\sigma_{r-\mathrm{s}-1}$ defines a bijection $\mathcal{L}_{1}^{\mathrm{s}+2} \cup \mathcal{M}_{1}^{\mathrm{s}+2} \rightarrow \mathcal{L}^{\mathrm{s}+1} \cup \mathcal{M}^{\mathrm{s}+1}$. The hypothesis (4.39b) implies $\mathcal{L}^{\mathrm{s}+1}\left(\mathrm{~T}_{\varphi}\right) \cup \mathcal{M}^{\mathrm{s}+1}\left(\mathrm{~T}_{\varphi}\right)=\left\{m-p \mid p=2^{\mathrm{s}}, 2^{\mathrm{s}}+1, \ldots, 2^{\mathrm{s}+1}-1\right\}$. These observations, taken with (4.40a), yield

$$
\mathcal{L}_{1}^{\mathrm{s}+2}\left(\mathrm{~T}_{\varphi}\right) \cup \mathcal{M}_{1}^{\mathrm{s}+2}\left(\mathrm{~T}_{\varphi}\right)=\left\{m-p \mid p=2^{\mathrm{s}+1}, 2^{\mathrm{s}+1}+1, \ldots, 3 \cdot 2^{\mathrm{s}}-1\right\} .
$$


Likewise, the assignment $\nu \mapsto \nu+\sigma_{r-s-1}+2\left(\sigma_{r-s}+\cdots+\sigma_{r-2}\right)+\sigma_{r-1}+\sigma_{r}$ defines a bijection $\mathcal{L}_{2}^{\mathbf{s}+2} \cup \mathcal{M}_{2}^{\mathrm{s}+2} \rightarrow \mathcal{F}^{\mathbf{s}} \cup \mathcal{G}^{\text {s. }}$. Taken with (4.39b), (4.40a) and (4.15), this yields

$$
\begin{aligned}
\mathcal{L}_{1}^{\mathrm{s}+2}\left(\mathrm{~T}_{\varphi}\right) \cup \mathcal{M}_{1}^{\mathrm{s}+2}\left(\mathrm{~T}_{\varphi}\right) & =\left\{\nu\left(\mathrm{T}_{\varphi}\right)-2^{\mathrm{s}}-2\left(2^{\mathrm{s}-1}+\cdots+2\right)-(1+3) \mid \nu \in \mathcal{F}^{\mathrm{s}} \cup \mathcal{G}^{\mathrm{s}}\right\} \\
& =\left\{\nu\left(\mathrm{T}_{\varphi}\right)-3 \cdot 2^{\mathrm{s}} \mid \nu \in \mathcal{F}^{\mathrm{s}} \cup \mathcal{G}^{\mathrm{s}}\right\} \\
& =\left\{m-p \mid p=3 \cdot 2^{\mathrm{s}}, 3 \cdot 2^{\mathrm{s}}+1, \ldots, 2^{\mathrm{s}+2}-1\right\} .
\end{aligned}
$$

Item (4.40b) now follows from (4.41). This yields (the first grading element of) (4.5).

\section{Exceptional groups}

Theorem 5.1. Let $G$ be a Hodge group with complex Lie algebra $\mathfrak{g}_{\mathbb{C}}=\mathfrak{e}_{6}(\mathbb{C}), \mathfrak{e}_{7}(\mathbb{C})$. Then $G$ does not admit a principal Hodge representation $(V, \varphi)$.

Theorem 5.2. Let $G$ be a Hodge group with complex Lie algebra $\mathfrak{g}_{\mathbb{C}}=\mathfrak{g}_{2}(\mathbb{C})$. Assume that $(V, \varphi)$ is a Hodge representation. Let $\mathrm{T}_{\varphi}$ be the associated grading element $(\S 2.3)$, and assume the normalization (2.2) holds. Then the Hodge representation is principal if and only if $V_{\mathbb{C}}=\mathbb{C}^{7}$ and $\mathrm{T}_{\varphi}=\mathrm{T}^{1}+\mathrm{T}^{2}$.

Proof of Theorem 5.1 for $\mathfrak{g}_{\mathbb{C}}=\mathfrak{e}_{6}(\mathbb{C})$. We argue by contradiction. Suppose a principal Hodge representation exists. Let $W_{1} \oplus \cdots \oplus W_{s}$ be a decomposition of $V_{\mathbb{R}}$ into irreducible $G_{\mathbb{R}}-$ modules. Let $U_{j}$ be the irreducible $G_{\mathbb{C}}$-module associated to $W_{j}$, as in $\S 2.4$. By $(2.13)$ and Theorem 2.14(e), the highest weight of $U_{j}$ is either $\omega_{1}$ or $\omega_{6}$. Moreover, $\omega_{1}^{*}=\omega_{6}$, so that $U_{j}$ is complex, $W_{j}(\mathbb{C})=W_{j} \otimes_{\mathbb{R}} \mathbb{C}=U_{j} \oplus U_{j}^{*}$, and $W_{j}(\mathbb{C}) \simeq W_{k}(\mathbb{C})$, for all $j, k$. The constraint $(2.10)$ that $V_{\mathbb{C}}=\oplus_{j} W_{j}(\mathbb{C})$ be weight multiplicity-free forces $s=1$. Thus, $V_{\mathbb{R}}$ is an irreducible $G_{\mathbb{R}}-$ module, and $V_{\mathbb{C}}=U \oplus U^{*}$.

Assume, without loss of generality, that $U$ is the irreducible $\mathfrak{e}_{6}$ representation of highest weight

$$
\omega_{1}=\frac{1}{3}\left(4 \sigma_{1}+3 \sigma_{2}+5 \sigma_{3}+6 \sigma_{4}+4 \sigma_{5}+2 \sigma_{6}\right) .
$$

Then, $\operatorname{dim} V_{\mathbb{C}}=2 \operatorname{dim}_{\mathbb{C}} U=54$. Therefore, (2.8) and (2.20) imply $53 / 2=\omega_{1}\left(\mathrm{~T}_{\varphi}\right)=\frac{1}{3}\left(4 n_{1}+\right.$ $\left.3 n_{2}+5 n_{3}+6 n_{4}+4 n_{5}+2 n_{6}\right)$. Equivalently,

$$
53 \cdot 3=2 \cdot\left(4 n_{1}+3 n_{2}+5 n_{3}+6 n_{4}+4 n_{5}+2 n_{6}\right) .
$$

This is not possible, as 2 does not divide $53 \cdot 3$.

Proof of Theorem 5.1 for $\mathfrak{g}_{\mathbb{C}}=\mathfrak{e}_{7}(\mathbb{C})$. We argue by contradiction. Suppose a principal Hodge representation exists. Let $W_{1} \oplus \cdots \oplus W_{s}$ be a decomposition of $V_{\mathbb{R}}$ into irreducible $G_{\mathbb{R}}$-modules. Let $U_{j}$ be the irreducible $G_{\mathbb{C}}$-module associated to $W_{j}$, as in $\S 2.4$. By (2.13) and Theorem 2.14(e), the highest weight of $U_{j}$ is $\omega_{7}$. Therefore, $W_{j}(\mathbb{C}) \simeq W_{k}(\mathbb{C})$, for all $j, k$. The constraint $(2.10)$ that $V_{\mathbb{C}}=\oplus_{j} W_{j}(\mathbb{C})$ be weight multiplicity-free forces $s=1$, and $V_{\mathbb{R}}$ is irreducible. Moreover, $\omega_{7}^{*}=\omega_{7}$, so that $U=U_{1}$ is either real or quaternionic. By (2.11), $V_{\mathbb{C}}=U$ is real.

The highest weight of $U$ is

$$
\omega_{7}=\frac{1}{2}\left(2 \sigma_{1}+3 \sigma_{2}+4 \sigma_{3}+6 \sigma_{4}+5 \sigma_{5}+4 \sigma_{6}+3 \sigma_{7}\right) .
$$

Since $\operatorname{dim}_{\mathbb{C}} U=56,(2.8)$ yields

$$
m=\frac{1}{2} 55 .
$$


The weights of $U$ with $|\lambda| \leq 4$ are $\omega_{7}=(0 \cdots 0)$,

$$
\omega_{7}-\sigma_{7}, \quad \omega_{7}-\left(\sigma_{6}+\sigma_{7}\right), \quad \omega_{7}-\left(\sigma_{5}+\sigma_{6}+\sigma_{7}\right), \quad \omega_{7}-\left(\sigma_{4}+\sigma_{5}+\sigma_{6}+\sigma_{7}\right) .
$$

All other weights are of the form $\omega_{7}-\left(\sigma_{4}+\sigma_{5}+\sigma_{6}+\sigma_{7}\right)-a^{i} \sigma_{i}$ with $0 \leq a^{i} \in \mathbb{Z}$. So, in order for (2.9) to hold, it is necessary that

$$
1=n_{7}, n_{6}, n_{5}, n_{4} .
$$

Observe that we have $\mathrm{T}_{\varphi}$-eigenvalues

$$
\left\{\lambda\left(\mathrm{T}_{\varphi}\right)|| \lambda \mid \leq 4\right\}=\{m-p \mid p=0,1,2,3,4\} .
$$

Next, the weights with $|\lambda|=5,6$ are

$$
\begin{aligned}
& \{\lambda \in \Lambda(U)|| \lambda \mid=5\}=\{(0101111),(0011111)\} \\
& \{\lambda \in \Lambda(U)|| \lambda \mid=6\}=\{(0111111),(1011111)\} .
\end{aligned}
$$

Therefore, to obtain the eigenvalue $m-5$, as required by (2.9), the weights (5.4) force either $n_{2}=1$ and $n_{3}>1$, or $n_{2}>1$ or $n_{3}=1$. Before proceeding to consider these two cases, it will be helpful to note that the remaining weights with $\lambda^{1}=0$ are

$$
\left\{\lambda \in \Lambda(U)\left|\lambda^{1}=0,\right| \lambda \mid>6\right\}=\{(0112111),(0112211),(0112221),(0112222)\} .
$$

Case 1: $n_{2}=1$ and $n_{3}>1$. Then the $\mathrm{T}_{\varphi}$-eigenvalues associated to weights with $\lambda^{1}=0$ are

$$
\left\{\lambda\left(\mathrm{T}_{\varphi}\right) \mid \lambda \in \Lambda(U), \lambda^{1}=0\right\}=\left\{m-p \mid p=0,1, \ldots, 5, n_{3}+4, n_{3}+5, \ldots, n_{3}+9\right\} .
$$

The largest eigenvalue $\lambda\left(\mathrm{T}_{\varphi}\right)$ with $\lambda^{1} \neq 0$ is $(1011111)\left(\mathrm{T}_{\varphi}\right)=m-\left(n_{1}+n_{3}+4\right)$. All other weights with $\lambda^{1} \neq 0$ are $\lambda \leq(1 \cdots 1)$, and so yield an eigenvalue $\lambda\left(\mathrm{T}_{\varphi}\right) \leq m-\left(5+n_{1}+n_{3}\right)$. Therefore, to realize the eigenvalue $m-6$, it is necessary that $n_{3}=2$. This then forces $n_{1}+6=(1011111)\left(\mathrm{T}_{\varphi}\right)=12$, so that $n_{1}=6$. However, if $\mathrm{T}_{\varphi}=n_{i} \mathrm{~T}^{i}=6 \mathrm{~T}^{1}+\mathrm{T}^{2}+2 \mathrm{~T}^{3}+$ $\mathrm{T}^{4}+\mathrm{T}^{5}+\mathrm{T}^{6}+\mathrm{T}^{7}$, then $\omega_{7}\left(\mathrm{~T}_{\varphi}\right)=\frac{1}{2}(2 \cdot 6+3 \cdot 1+4 \cdot 2+6 \cdot 1+5 \cdot 1+4 \cdot 1+3 \cdot 1)=\frac{1}{2} 41 \neq m$, a contradiction of (2.20) and (5.3).

Case 2: $n_{2}>1$ and $n_{3}=1$. In this case, we have

$$
\left\{\lambda\left(\mathrm{T}_{\varphi}\right) \mid \lambda \in \Lambda(U), \lambda^{1}=0\right\}=\left\{m-p \mid p=0,1, \ldots, 5, n_{2}+4, n_{2}+5, \ldots, n_{2}+9\right\} .
$$

The largest eigenvalue $\lambda\left(\mathrm{T}_{\varphi}\right)$ with $\lambda^{1} \neq 0$ is $(1011111)\left(\mathrm{T}_{\varphi}\right)=m-\left(n_{1}+5\right)$. All other weights with $\lambda^{1} \neq 0$ are $\lambda \leq(1 \cdots 1)$, and so yield an eigenvalue $\lambda\left(\mathrm{T}_{\varphi}\right) \leq m-\left(5+n_{1}+n_{2}\right)$. Therefore, in order to realize the eigenvalue $m-6$, we must have either $n_{1}=1$ or $n_{2}=2$.

Case 2.a: $n_{2}>1, n_{3}=1$ and $n_{1}=1$. If $n_{1}=1$, then we must have $n_{2}=3$ in order to realize the eigenvalue $m-7=(0101111)\left(\mathrm{T}_{\varphi}\right)$. However, if $\mathrm{T}=n_{i} \mathrm{~T}^{i}=\mathrm{T}^{1}+3 \mathrm{~T}^{2}+\mathrm{T}^{3}+\mathrm{T}^{4}+\mathrm{T}^{5}+\mathrm{T}^{6}+\mathrm{T}^{7}$, then $\omega_{7}\left(\mathrm{~T}_{\varphi}\right)=\frac{1}{2}(2 \cdot 1+3 \cdot 3+4 \cdot 1+6 \cdot 1+5 \cdot 1+4 \cdot 1+3 \cdot 1)=\frac{1}{2} 33 \neq m$, a contradiction of $(2.20)$ and $(5.3)$.

Case 2.b: $n_{2}=2$ and $n_{3}=1$. If $n_{2}=2$, then in order to avoid multiplicity, as required by (2.7), and realize the eigenvalue $m-12$ we must have $n_{1}=7$. In this case, $\mathrm{T}=n_{i} \mathrm{~T}^{i}=7 \mathrm{~T}^{1}+$ $2 \mathrm{~T}^{2}+\mathrm{T}^{3}+\mathrm{T}^{4}+\mathrm{T}^{5}+\mathrm{T}^{6}+\mathrm{T}^{7}$, so that $\omega_{7}\left(\mathrm{~T}_{\varphi}\right)=\frac{1}{2}(2 \cdot 7+3 \cdot 2+4 \cdot 1+6 \cdot 1+5 \cdot 1+4 \cdot 1+3 \cdot 1)=\frac{1}{2} 42 \neq m$. Again, this contradicts (2.20) and (5.3). 
Proof of Theorem 5.2. Suppose $(V, \varphi)$ is a principal Hodge representation of $G$. Let $W_{1} \oplus \cdots \oplus W_{s}$ be a decomposition of $V_{\mathbb{R}}$ into irreducible $G_{\mathbb{R}}$-modules. Let $U_{j}$ be the irreducible $G_{\mathbb{C}}$-module associated to $W_{j}$, as in $\S 2.4$. By $(2.13)$ and Theorem 2.14(e), the highest weight of $U_{j}$ is $\omega_{1}$. Therefore, $W_{j}(\mathbb{C}) \simeq W_{k}(\mathbb{C})$, for all $j, k$. The constraint $(2.10)$ that $V_{\mathbb{C}}=\oplus_{j} W_{j}(\mathbb{C})$ be weight multiplicity-free forces $s=1$. Therefore, $V_{\mathbb{R}}$ is an irreducible $G_{\mathbb{R}^{-}}$-module. Moreover, $\omega_{1}^{*}=\omega_{1}$, so that $U=U_{1}$ is either real or quaternionic. By (2.11), $U$ must be real. Thus, $V_{\mathbb{C}}=U=\mathbb{C}^{7}$.

The highest weight is

$$
\omega_{1}=2 \sigma_{1}+\sigma_{2} \text {. }
$$

Since $U$ is self-dual, it is real or quaternionic, cf. $\S 2.4$. If $V$ is principal, then (2.11) implies $U$ is real and $V_{\mathbb{C}}=U$. So the weights of $V_{\mathbb{C}}$ are

$$
\begin{aligned}
\Lambda(U)=\{ & \omega_{1}, \omega_{1}-\sigma_{1}, \omega_{1}-\left(\sigma_{1}+\sigma_{2}\right), \omega_{1}-\left(2 \sigma_{1}+\sigma_{2}\right), \\
& \left.\omega_{1}-\left(3 \sigma_{1}+\sigma_{2}\right), \omega_{1}-\left(3 \sigma_{1}+2 \sigma_{2}\right), \omega_{1}-\left(4 \sigma_{1}+2 \sigma_{2}\right)\right\} .
\end{aligned}
$$

In particular, the weights include $\omega_{1}, \omega_{1}-\sigma_{1}, \omega_{1}-\left(\sigma_{1}+\sigma_{2}\right)$, and all other weights are of the form $\omega_{1}-\left(a \sigma_{1}+b \sigma_{2}\right)$ with $0<a, b \in \mathbb{Z}$ and $a+b>2$. Given (2.9), this forces $\mathrm{T}_{\varphi}=\mathrm{T}^{1}+\mathrm{T}^{2}$, which yields a decomposition $V_{\mathbb{C}}=V_{3} \oplus V_{2} \oplus V_{1} \oplus V_{0} \oplus V_{-1} \oplus V_{-2} \oplus V_{-3}$ with all Hodge numbers equal to one.

Finally, we note that $(2.3)$ yields $\mathrm{T}^{\mathrm{cpt}}=0$. So $\mu\left(\mathrm{T}^{\mathrm{cpt}}\right)=0$, and $U$ is real by $(2.4)$.

\section{Special linear Hodge groups}

Assume throughout this section that

$$
G \text { is a } \mathbb{Q} \text {-algebraic group with Lie algebra } \mathfrak{g}_{\mathbb{C}} \simeq \mathfrak{s l}_{r+1} \mathbb{C} \text {. }
$$

The fundamental weights of $\mathfrak{g}_{\mathbb{C}}=\mathfrak{s l}_{r+1} \mathbb{C}$ are

$$
\begin{aligned}
\omega_{k}= & \frac{r+1-k}{r+1}\left[\sigma_{1}+2 \sigma_{2}+\cdots+(k-1) \sigma_{k-1}\right]+\frac{k(r+1-k)}{r+1} \sigma_{k} \\
& +\frac{k}{r+1}\left[(r-k) \sigma_{k+1}+(r-k-1) \sigma_{k+2}+\cdots+\sigma_{r}\right],
\end{aligned}
$$

$1 \leq k \leq r$. If $U$ is the irreducible $\mathfrak{g}_{\mathbb{C}^{-}}$module of highest weight $\omega_{k}$, then the highest weight of the dual $U^{*}$ is

$$
\omega_{k}^{*}=\omega_{r+1-k} .
$$

In particular, in the notation of Lemma $2.22, k^{*}=r+1-k$.

6.1. Restrictions on principal Hodge representations. In this section we describe some simple numerical constraints on principal Hodge representations. Among those are the restriction that the rank of $G$ be odd. The cases of rank $r=1,3,5$ are addressed in Propositions $6.12,6.13$ and 6.16 ; a examples of rank seven and nine are considered in $\S 6.5$.

By (6.1),

$$
\omega_{k}\left(\mathrm{~T}_{\varphi}\right) \in \frac{1}{r+1} \mathbb{Z} .
$$

Lemma 6.4. Suppose that $G$ admits a principal Hodge representation. Then $r+1$ is even. 
Proof of Lemma 6.4. Let $V_{\mathbb{R}}=W_{1} \oplus \cdots \oplus W_{s}$ be a decomposition into irreducible $G_{\mathbb{R}^{-}}$ modules. Let $U_{j}$ be the irreducible $G_{\mathbb{C}^{-}}$module associated to $W_{j}$ as in $\S 2.4$. If one of the $U_{j}$ is self-dual, then $r+1$ is necessarily even by (6.2).

Assume none of the $U_{j}$ are self-dual. Then, by $\S 2.4, W_{j}(\mathbb{C})=U_{j} \oplus U_{j}^{*}$, for all $1 \leq j \leq s$. In particular, each $W_{j}(\mathbb{C})$ is of even dimension. It follows that $V_{\mathbb{C}}=\oplus_{j} W_{j}(\mathbb{C})$ is of even dimension. So (2.8) implies

$$
m=\frac{2 a-1}{2}
$$

for some $0<a \in \mathbb{Z}$.

Let $\mu_{j}$ denote the highest weight of $U_{j}$. By (2.20), there exists $1 \leq j \leq s$ such that the largest eigenvalue $m=\mu_{j}\left(\mathrm{~T}_{\varphi}\right)$. By $(2.10)$, the representation $U_{j}$ is weight multiplicity-free. By Theorem 2.14(a), the weight $\mu_{j}$ is necessarily of the form $p \omega_{k}$ for some $p$ and $k$. From (2.20) and (6.3), we see that

$$
m=\frac{b}{r+1}
$$

for some $0<b \in \mathbb{Z}$. Together (6.5) and (6.6) yield $(r+1)(2 a-1)=2 b$, implying 2 divides $r+1$.

Lemma 6.7. Suppose that $r>1$ and $G$ admits a principal Hodge representation $(V, \varphi)$ with $V_{\mathbb{C}}=\operatorname{Sym}^{p}\left(\mathbb{C}^{r+1}\right) \oplus \operatorname{Sym}^{p}\left(\mathbb{C}^{r+1}\right)^{*}$. Then $(r+1) ! \equiv 0 \bmod 2 p$, and $2 p \not \equiv 0 \bmod r+1$.

Proof of Lemma 6.7, Part 1. First, we prove that $(r+1) ! \in 2 p \mathbb{Z}$. Note that

$$
\operatorname{dim}_{\mathbb{C}} \operatorname{Sym}^{p}\left(\mathbb{C}^{r+1}\right)=\left(\begin{array}{c}
p+r \\
r
\end{array}\right)=\frac{(p+r)(p+r-1) \cdots(p+1)}{r !} .
$$

The highest weight of $\operatorname{Sym}^{p}\left(\mathbb{C}^{r+1}\right)$ is $\mu=p \omega_{1}$. By (2.8), (2.20) and (6.3),

$$
\frac{1}{2}\left[2 \frac{(p+r)(p+r-1) \cdots(p+1)}{r !}-1\right] \in \frac{p}{r+1} \mathbb{Z}
$$

Equivalently,

$$
\frac{1}{2}(r+1)[2(p+r)(p+r-1) \cdots(p+1)-r !] \in p r ! \mathbb{Z} .
$$

Note that $(p+r)(p+r-1) \cdots(p+1) \equiv r ! \bmod p$. In particular, there exists $q \in \mathbb{Z}$ such that

$$
\frac{1}{2}(r+1)(2 r !+p q-r !)=\frac{1}{2}(r+1) !+\frac{1}{2}(r+1) p q \in p r ! \mathbb{Z} .
$$

so that $(r+1) ! \in 2 p \mathbb{Z}$.

Proof of Lemma 6.7, Part 2. Now we prove that $2 p \not \equiv 0$ modulo $r+1$. The weights of $\mathbb{C}^{r+1}$ and $U=\operatorname{Sym}^{p}\left(\mathbb{C}^{r+1}\right)$, as a $\mathfrak{g}_{\mathbb{C}^{-} \text {modules, are }}$

$$
\begin{aligned}
\Lambda\left(\mathbb{C}^{r+1}\right) & =\left\{\nu_{i} \stackrel{\text { dfn }}{=} \omega_{1}-\left(\sigma_{1}+\cdots+\sigma_{i-1}\right) \mid 1 \leq i \leq r+1\right\}, \\
\Lambda(U) & =\left\{\nu_{i_{1}}+\cdots+\nu_{i_{p}} \mid 1 \leq i_{1} \leq \cdots \leq i_{p} \leq r+1\right\} .
\end{aligned}
$$

Note that

$$
\nu_{1}+\cdots+\nu_{r+1}=0
$$


By Lemma 6.4, $r+1=2 s$, for some $s \in \mathbb{Z}$. Then (6.9) implies

$$
\nu_{1}+\cdots+\nu_{s}=-\left(\nu_{s+1}+\cdots+\nu_{r+1}\right) .
$$

Arguing by contradiction, suppose that $r+1$ divides $2 p$. We will consider two cases: $2 p=2 t(r+1)$ and $2 p=(2 t+1) \cdot(r+1)$, for some $t \in \mathbb{Z}$.

$\circ$ First, suppose that $2 p=2 t(r+1)$. Then $p=t(r+1)$, and (6.8) and (6.9) imply $0=t\left(\nu_{1}+\cdots+\nu_{r+1}\right) \in \Lambda(U)$. This contradicts $(2.12)$.

○ Next, suppose that $2 p=(2 t+1) \cdot(r+1)$, so that $p=(2 t+1) s$. Then $(6.8),(6.9)$ and (6.10) imply that both

$$
\begin{aligned}
\nu_{1}+\cdots+\nu_{s} & =\left(\nu_{1}+\cdots+\nu_{s}\right)+t\left(\nu_{1}+\cdots+\nu_{r+1}\right), \text { and } \\
-\left(\nu_{1}+\cdots+\nu_{s}\right) & =\left(\nu_{s+1}+\cdots+\nu_{r+1}\right) \\
& =\left(\nu_{s+1}+\cdots+\nu_{r+1}\right)+t\left(\nu_{1}+\cdots+\nu_{r+1}\right)
\end{aligned}
$$

are weights of $U$. Again, this contradicts (2.12).

Lemma 6.11. Suppose that $1 \leq k<\frac{1}{2}(r+1)$ and that $2 k \neq \frac{1}{2}(r+1)$. Assume $G$ admits a principal Hodge representation $(V, \varphi)$ with $V_{\mathbb{R}}$ irreducible and $V_{\mathbb{C}}=\bigwedge^{2 k}\left(\mathbb{C}^{r+1}\right) \oplus \bigwedge^{2 k}\left(\mathbb{C}^{r+1}\right)^{*}$. Then $r+1 \equiv 0 \bmod 4$.

Remark. From (6.2), we see that the condition $2 k \neq \frac{1}{2}(r+1)$ implies $U=\bigwedge^{2 k}\left(\mathbb{C}^{r+1}\right)$ is not self-dual. By $\S 2.4$, the representation $V_{\mathbb{C}}=U \oplus U^{*}$ is complex.

Proof. We argue by contradiction. Let $\mathrm{T}_{\varphi}=n_{i} \mathrm{~T}^{i}$ be the grading element associated to the Hodge representation. We have $\operatorname{dim}_{\mathbb{C}} V_{\mathbb{C}}=2 a$ for $a=\operatorname{dim}_{\mathbb{C}} U$. By Lemma $6.4, r+1=2 s$ for some $s \in \mathbb{Z}$. By (2.8) and (2.20),

$$
\begin{aligned}
\frac{1}{2}(2 a-1) \stackrel{ }{=} & \omega_{2 k}\left(\mathrm{~T}_{\varphi}\right) \\
\stackrel{(6.1)}{=} & \frac{(s-k)}{s}\left(n_{1}+2 n_{2}+\cdots+(2 k-1) n_{2 k-1}\right)+\frac{2 k(s-k)}{s} n_{2 k} \\
& +\frac{k}{s}\left((r-2 k) n_{2 k+1}+(r-2 k-1) n_{2 k+2}+\cdots+n_{r}\right) .
\end{aligned}
$$

Multiplying through by $2 s$ yields $(2 a-1) s \in 2 \mathbb{Z}$. Thus $r+1=2 s \equiv 0 \bmod 4$.

6.2. The rank one case. The irreducible representations of $\mathfrak{s l}_{2} \mathbb{C}$ are $\operatorname{Sym}^{p} \mathbb{C}^{2}$; they are of highest weight $\mu=p \omega_{1}=\frac{1}{2} p \sigma_{1}$.

Proposition 6.12. Let $G$ be a Hodge group with complex Lie algebra $\mathfrak{g}_{\mathbb{C}}=\mathfrak{s l}_{2} \mathbb{C}$. Assume that $(V, \varphi)$ is a Hodge representation with the property that $V_{\mathbb{C}}=\operatorname{Sym}^{p} \mathbb{C}^{2}$, and satisfying the normalization (2.2). Then $V$ is principal if and only if $\mathrm{T}_{\varphi}=\mathrm{T}_{1}$.

Proof. Let $U$ be the irreducible $G_{\mathbb{C}}$-module associated to $V_{\mathbb{R}}$ by $\S 2.4$. Let $\mu=p \omega_{1}$ be the highest weight of $U$. By Theorem 2.14(a), $U=\mathrm{Sym}^{p} \mathbb{C}^{2}$ is multiplicity-free as required by (2.13).

Since $U$ is self-dual, it is either real or quaternionic, cf. $\S 2.4$. By (2.11), if $V$ is principal, then $U$ is real; therefore, $V_{\mathbb{C}}=U$. By Lemma $2.22(\mathrm{a}), n_{1}=1$; so the grading element is necessarily of the form $\mathrm{T}_{\varphi}=\mathrm{T}^{1}$. 
ROBLES

The weights of $V_{\mathbb{C}}$ are $\Lambda\left(V_{\mathbb{C}}\right)=\left\{\frac{1}{2}(p-i) \sigma_{1} \mid 0 \leq i \leq p\right\}$. In particular, (2.9) holds. To conclude that the Hodge representation is principal, it remains to confirm that $V_{\mathbb{C}}$ is a real (rather than quaternionic) $G_{\mathbb{R}^{-}}$module: definition (2.3) yields $\mathrm{T}^{\mathrm{cpt}}=0$, so that $\mu\left(\mathrm{T}^{\mathrm{cpt}}\right)=0$ is even, as required by (2.4) and (2.11).

\subsection{The rank three case.}

Proposition 6.13. Let $G$ be a Hodge group with complex Lie algebra $\mathfrak{g}_{\mathbb{C}}=\mathfrak{s l}_{4} \mathbb{C}$. Assume that $(V, \varphi)$ is a Hodge representation with the property that $V_{\mathbb{R}}$ is an irreducible $G_{\mathbb{R}}$-module. Let $\mathrm{T}_{\varphi}$ be the associated grading element (\$2.3), and assume the normalization (2.2) holds. Then the Hodge representation is principal if and only if $V_{\mathbb{C}}=\mathbb{C}^{4} \oplus\left(\mathbb{C}^{4}\right)^{*}$ and $\mathrm{T}_{\varphi}$ is one of

$$
3 \mathrm{~T}^{1}+2 \mathrm{~T}^{2}+\mathrm{T}^{1} \quad \text { or } \quad \mathrm{T}^{1}+2 \mathrm{~T}^{2}+3 \mathrm{~T}^{3} .
$$

Proof. Let $U$ be the irreducible $G_{\mathbb{C}^{-}}$module of highest weight $\mu$ associated to $V_{\mathbb{R}}$ as in $\S 2.4$. By (2.13), $U$ is necessarily weight multiplicity-free. By Theorem 2.14(a), the highest weight of $U$ is either $\mu=p \omega_{1}$ (or the symmetric case $\mu=p \omega_{3}$ ); or $\mu=\omega_{2}$.

(I) Let's begin with the case that $\mu=p \omega_{1}$. Then $U=\operatorname{Sym}^{p} \mathbb{C}^{4}$ and $V_{\mathbb{C}}=U \oplus U^{*}$. We have $r+1=4=2^{2}$ and $(r+1) !=24=3 \cdot 2^{3}$. By Lemma $6.7, p \mid 3 \cdot 2^{2}$, but $2 \neq p$. Therefore, $p=1,3$.

Observe that

$$
m \stackrel{(2.20)}{=} p \omega_{1}\left(\mathrm{~T}_{\varphi}\right)=\frac{1}{4}\left(3 n_{1}+2 n_{2}+n_{3}\right), \quad \text { and } \quad 0 \stackrel{(*)}{<} m-m^{*}=p \frac{1}{2}\left(n_{1}-n_{3}\right),
$$

where the inequality $(*)$ is due to Lemma $2.22(\mathrm{~b})$. From $(2.9)$ we see that any two eigenvalues differ by an integer; therefore, $0<m-m^{*} \in \mathbb{Z}$. Since $p=1,3$, this implies $n_{1}-n_{3} \in 2 \mathbb{Z}$. Then (2.17) yields $1 \leq n_{3} \leq n_{1}-2$. In particular, $n_{1} \geq 3$. By Lemma $2.22(\mathrm{~b})$, we must have $n_{3}=1$ and $m-m^{*}=1$, the latter yielding $p=1$ and $n_{1}=3$. From $\operatorname{dim} V_{\mathbb{C}}=8$ and (2.8) we see that $\frac{1}{2} 7=m$, yielding $n_{2}=2$. Thus, $\mathrm{T}_{\varphi}=3 \mathrm{~T}^{1}+2 \mathrm{~T}^{2}+\mathrm{T}^{1}$ is the first grading element of the proposition.

If, on the other hand, we take $\mu=p \omega_{3}$, then a similar argument again yields $p=1$ and $\mathrm{T}_{\varphi}$ is the second grading element of the proposition. In either case $\left(\mu=\omega_{1}\right.$ or $\left.\mu=\omega_{3}\right)$, it is easily checked that (2.9) holds, using the fact that the weights of $V_{\mathbb{C}}$ are

$$
\begin{aligned}
& \left\{\omega_{1}, \omega_{1}-\sigma_{1}, \omega_{1}-\left(\sigma_{1}+\sigma_{2}\right), \omega_{1}-\left(\sigma_{1}+\sigma_{2}+\sigma_{3}\right)\right\} \cup \\
& \left\{\omega_{3}, \omega_{3}-\sigma_{3}, \omega_{3}-\left(\sigma_{3}+\sigma_{2}\right), \omega_{3}-\left(\sigma_{3}+\sigma_{2}+\sigma_{1}\right)\right\}
\end{aligned}
$$

(II) Next, consider the case that $\mu=\omega_{2}$. We have $U=\bigwedge^{2} \mathbb{C}^{6}$. Since $U$ is self-dual, $U$ is either real or quaternionic (§2.4). By (2.11), if $V$ is principal, then $U$ is necessarily real, so that $V_{\mathbb{C}}=U$. By Lemma $2.22(\mathrm{a}), n_{2}=1$. The highest weight of the irreducible $V_{\mathbb{C}}$ is $\omega_{2}=\frac{1}{2}\left(\sigma_{1}+2 \sigma_{2}+\sigma_{3}\right)$. By $(2.8)$ and $(2.20)$, we have

$$
\frac{1}{2} 5=\frac{1}{2}\left(n_{1}+2 n_{2}+n_{3}\right)=1+\frac{1}{2}\left(n_{1}+n_{3}\right),
$$

so that $n_{1}+n_{3}=3$. From (2.17), we see that either $n_{1}=1$ and $n_{3}=2$, or $n_{1}=1$ and $n_{3}=2$. Thus,

$$
\mathrm{T}_{\varphi}=\mathrm{T}^{1}+\mathrm{T}^{2}+2 \mathrm{~T}^{3} \quad \text { or } \quad \mathrm{T}_{\varphi}=2 \mathrm{~T}^{1}+\mathrm{T}^{2}+\mathrm{T}^{3} .
$$


For the first grading element, we have $\mathrm{T}^{\mathrm{cpt}}=2 \mathrm{~T}^{3}$, cf. (2.3). So $\mu\left(\mathrm{T}^{\mathrm{cpt}}\right)=\omega_{2}\left(2 \mathrm{~T}^{3}\right)=1$ implying $U$ is quaternionic, a contradiction of (2.11). Similarly, for the second grading element, $\mathrm{T}^{\mathrm{cpt}}=2 \mathrm{~T}^{1}$. So $\mu\left(\mathrm{T}^{\mathrm{cpt}}\right)=\omega_{2}\left(2 \mathrm{~T}^{1}\right)=1$ and $U$ is quaternionic, again contradicting (2.11). Therefore, there exists no principal Hodge representation $(V, \varphi)$ with $V=\bigwedge^{2} \mathbb{C}^{4}$.

Remark 6.15. With respect to part (II) of the proof above, observe that the weights of $\bigwedge^{2} \mathbb{C}^{4}$ are

$$
\text { (000), (010), (110), (011), (111), (121). }
$$

(Above, we utilize the notation $\left(\lambda^{1} \lambda^{2} \lambda^{3}\right)=\omega_{2}-\lambda^{i} \sigma_{i}$ introduced in §2.7.) From this, it is easily checked that both the grading elements (6.14) yield eigenspace decompositions of $\wedge^{2} \mathbb{C}^{4}$ with multiplicity-free eigenvalues $\pm\left\{\frac{1}{2}, \frac{1}{2} 3, \frac{1}{2} 5\right\}$. Thus, the following holds: Let $G$ be a Hodge group with complex Lie algebra $\mathfrak{g}_{\mathbb{C}}=\mathfrak{s l}_{4} \mathbb{C}$. Assume that $(V, \varphi)$ is a Hodge representation with the property that $V_{\mathbb{R}}$ is irreducible and $V_{\mathbb{C}}=\left(\bigwedge^{2} \mathbb{C}^{4}\right) \oplus\left(\bigwedge^{2} \mathbb{C}^{4}\right)^{*}$. (That is, $U=\wedge^{2} \mathbb{C}^{4}$ is quaternionic.) Then the Hodge numbers are $\mathbf{h}=(2,2, \ldots, 2,2)$ if only if $\mathrm{T}_{\varphi}$ is one of $(6.14)$.

\subsection{The rank five case.}

Proposition 6.16. Let $G$ be a Hodge group with complex Lie algebra $\mathfrak{g}_{\mathbb{C}}=\mathfrak{s l}_{6} \mathbb{C}$. Assume that $(V, \varphi)$ is a Hodge representation with the property that $V_{\mathbb{R}}$ is an irreducible $G_{\mathbb{R}}$-module. Let $\mathrm{T}_{\varphi}$ be the associated grading element (§2.3), and assume the normalization (2.2) holds. Then the Hodge representation is principal if and only if either: (a) $V_{\mathbb{C}}=\mathbb{C}^{6} \oplus\left(\mathbb{C}^{6}\right)^{*}$ and

$$
\begin{aligned}
& \mathrm{T}_{\varphi}=2 \mathrm{~T}^{1}+4 \mathrm{~T}^{2}+\mathrm{T}^{3}+\mathrm{T}^{4}+2 \mathrm{~T}^{5}, \quad \text { or } \\
& \mathrm{T}_{\varphi}=2 \mathrm{~T}^{1}+\mathrm{T}^{2}+\mathrm{T}^{3}+4 \mathrm{~T}^{4}+2 \mathrm{~T}^{5} ;
\end{aligned}
$$

or (b) $V_{\mathbb{C}}=\bigwedge^{3} \mathbb{C}^{6}$ and

$$
\begin{aligned}
& \mathrm{T}_{\varphi}=3 \mathrm{~T}^{1}+2 \mathrm{~T}^{2}+\mathrm{T}^{3}+\mathrm{T}^{4}+7 \mathrm{~T}^{5}, \quad \text { or } \\
& \mathrm{T}_{\varphi}=7 \mathrm{~T}^{1}+\mathrm{T}^{2}+\mathrm{T}^{3}+2 \mathrm{~T}^{4}+3 \mathrm{~T}^{5} .
\end{aligned}
$$

Proof. Let $U$ be the irreducible $G_{\mathbb{C}^{-}}$module of highest weight $\mu$ associated to $V_{\mathbb{R}}$ as in $\S 2.4$. By (2.13), $U$ is necessarily weight multiplicity-free. By Theorem 2.14(a), the highest weight of $U$ is either $\mu=p \omega_{1}, p \omega_{r}$, or $\mu=\omega_{k}$ with $k=2,3,4$. The cases $\mu=p \omega_{1}$ and $\mu=p \omega_{r}$ are symmetric, as are the cases $\mu=\omega_{2}$ and $\mu=\omega_{4}$. So it suffices to consider the three cases $\mu=p \omega_{1}, \omega_{2}, \omega_{3}$.

(I) Let's begin with the case that

$$
\mu=p \omega_{1}=p \frac{1}{6}\left(5 \sigma_{1}+4 \sigma_{2}+3 \sigma_{3}+2 \sigma_{4}+\sigma_{5}\right) .
$$

Then $U=\operatorname{Sym}^{p} \mathbb{C}^{6} \not U^{*}$ and $V_{\mathbb{C}}=U \oplus U^{*}$. By Lemma 6.7, $2 p$ divides 6!; equivalently, $p$ divides $5 \cdot 3^{2} \cdot 2^{3}$. The lemma also asserts that 6 does not divide $2 p$. Therefore,

$$
p=5^{a} \cdot 2^{b}, \text { with } 0 \leq a \leq 1 \text { and } 0 \leq b \leq 3 .
$$

By (2.8) and (2.20),

$$
2 \operatorname{dim}_{\mathbb{C}} U-1=\frac{1}{3} 5^{a} 2^{b}\left(5 n_{1}+4 n_{2}+3 n_{3}+2 n_{4}+n_{5}\right)
$$

Since the left-hand side of (6.18) is odd, it is necessarily the case that $b=0$. Therefore, $p=1,5$. 
Suppose that $p=5$. Then $\operatorname{dim}_{\mathbb{C}} U=\left(\begin{array}{c}10 \\ 5\end{array}\right)=6^{2} \cdot 7$. Since $2 \operatorname{dim}_{\mathbb{C}} U-1=2 \cdot 6^{2} \cdot 7-1=503$ is not divisible by $5,(6.18)$ forces $b=0$. Thus, $p=1$, and we have $V_{\mathbb{C}}=\mathbb{C}^{6} \oplus\left(\mathbb{C}^{6}\right)^{*}$. With the assistance of mathematical software (such as the representation theory package LiE) one may confirm that (2.9) holds if and only if $\mathrm{T}_{\varphi}$ is the first grading element of (6.17a).

A nearly identical argument with $\mu=p \omega_{5}$ in place of $\mu=p \omega_{1}$ yields $p=1$ and $\mathrm{T}_{\varphi}$ is the second grading element of (6.17a).

(II) Next, consider the case that

$$
\mu=\omega_{2}=\frac{1}{3}\left(2 \sigma_{1}+4 \sigma_{2}+3 \sigma_{3}+2 \sigma_{4}+\sigma_{5}\right) .
$$

We have $U=\wedge^{2} \mathbb{C}^{6} \not \bigwedge^{4}\left(\mathbb{C}^{6}\right)=U^{*}$ and $V_{\mathbb{C}}=U \oplus U^{*}$. From $(2.8)$ and $\operatorname{dim}_{\mathbb{C}} U=\left(\begin{array}{l}6 \\ 2\end{array}\right)=15$, we obtain

Then (2.20) implies

$$
m=\frac{1}{2} 29 .
$$

$$
\frac{1}{2} 29=\frac{1}{3}\left(2 n_{1}+4 n_{2}+3 n_{3}+2 n_{4}+n_{5}\right) .
$$

This is not possible, as 2 does not divide $29 \cdot 3$.

(III) Finally, we consider the case that

$$
\mu=\omega_{3}=\frac{1}{2}\left(\sigma_{1}+2 \sigma_{2}+3 \sigma_{3}+2 \sigma_{4}+\sigma_{5}\right) .
$$

We have $U=\wedge^{3} \mathbb{C}^{6} \simeq U^{*}$. By $\S 2.4, U$ is either real or quaternionic. By $(2.11), U$ is necessarily real, so that $V_{\mathbb{C}}=U$. Again, with the assistance of $\mathrm{LiE}$, one may confirm that (2.9) holds if and only if (6.17b) holds.

For the first grading element of $(6.17 \mathrm{~b})$, we have $\mathrm{T}^{\mathrm{cpt}}=2 \mathrm{~T}^{2}$, cf. (2.3). Therefore, $\mu\left(\mathrm{T}^{\mathrm{cpt}}\right)=2$, and $U$ is a real representation of $G_{\mathbb{R}}$ by $(2.4)$, and as required by (2.11). Likewise, for the second grading element of $(6.17 \mathrm{~b})$, we have $\mathrm{T}^{\mathrm{cpt}}=2 \mathrm{~T}^{4}$ and $\mu\left(\mathrm{T}^{\mathrm{cpt}}\right)=2$; again, $U$ is a real representation.

\subsection{The standard representation: examples.}

Example (Rank seven). Let $G$ be a Hodge group with complex Lie algebra $\mathfrak{g}_{\mathbb{C}}=\mathfrak{s l}_{8} \mathbb{C}$. Assume that $(V, \varphi)$ is a Hodge representation with the property that $V_{\mathbb{R}}$ is an irreducible $G_{\mathbb{R}}$-module, and $V_{\mathbb{C}}=\mathbb{C}^{8} \oplus\left(\mathbb{C}^{8}\right)^{*}$. Let $\mathrm{T}_{\varphi}$ be the associated grading element ( $(2.3)$, and let $\mu=\omega_{1}$ be the highest weight of $\mathbb{C}^{8}$. Assume the normalizations (2.2) and (2.19) hold. A computation with LiE indicates that the Hodge representation is principal if and only if $\mathrm{T}_{\varphi}=n_{i} \mathrm{~T}^{i}$ is given by one of

$$
\begin{aligned}
\left(n_{1}, n_{2}, \ldots, n_{8}\right)= & (1,5,1,3,1,1,1),(2,3,2,2,2,1,2), \\
& (3,1,3,2,1,3,1),(3,2,1,2,3,2,1) .
\end{aligned}
$$

Suppose, on the other hand, that $\mu=\omega_{7}$ and the normalizations (2.2) and (2.19) hold. Then $\mathrm{T}_{\varphi}=\sum_{i} n_{8-i} \mathrm{~T}^{i}=n_{7} \mathrm{~T}^{1}+n_{6} \mathrm{~T}^{2}+\cdots+n_{2} \mathrm{~T}^{6}+n_{1} \mathrm{~T}^{7}$, where $\left(n_{1}, \ldots, n_{7}\right)$ is one of the four above.

Example (Rank nine). Let $G$ be a Hodge group with complex Lie algebra $\mathfrak{g}_{\mathbb{C}}=\mathfrak{s l}_{10} \mathbb{C}$. Assume that $(V, \varphi)$ is a Hodge representation with the property that $V_{\mathbb{R}}$ is an irreducible $G_{\mathbb{R}}$-module, and $V_{\mathbb{C}}=\mathbb{C}^{10} \oplus\left(\mathbb{C}^{10}\right)^{*}$. Let $\mathrm{T}_{\varphi}$ be the associated grading element $(\S 2.3)$, and let $\mu=\omega_{1}$ be the highest weight of $\mathbb{C}^{10}$. Assume the normalizations (2.2) and (2.19) hold. 
A computation with LiE indicates that the Hodge representation is principal if and only if $\mathrm{T}_{\varphi}=n_{i} \mathrm{~T}^{i}$ is given by one of

$$
\begin{aligned}
\left(n_{1}, n_{2}, \ldots, n_{10}\right)= & (1,2,6,2,1,1,1,1,2),(1,3,4,2,2,1,1,2,1), \\
& (1,4,2,3,1,2,2,1,1),(2,1,5,2,2,1,1,1,3), \\
& (2,2,3,3,1,2,1,2,2),(2,3,1,4,1,1,3,1,2), \\
& (2,4,1,1,1,5,1,1,2),(3,1,2,4,1,1,2,3,1), \\
& (3,2,2,1,1,4,2,2,1),(4,1,1,2,1,3,4,1,1) .
\end{aligned}
$$

Suppose, on the other hand, that $\mu=\omega_{9}$ and the normalizations (2.2) and (2.19) hold. Then $\mathrm{T}_{\varphi}=\sum_{i} n_{10-i} \mathrm{~T}^{i}=n_{9} \mathrm{~T}^{1}+n_{8} \mathrm{~T}^{2}+\cdots+n_{2} \mathrm{~T}^{8}+n_{1} \mathrm{~T}^{9}$, where $\left(n_{1}, \ldots, n_{9}\right)$ is one of the ten above.

\section{REFERENCES}

[1] Andreas Čap and Jan Slovák. Parabolic geometries. I, volume 154 of Mathematical Surveys and Monographs. American Mathematical Society, Providence, RI, 2009. Background and general theory.

[2] Michael Dettweiler and Stefan Reiter. Rigid local systems and motives of type $G_{2}$. Compos. Math., 146(4):929-963, 2010. With an appendix by Michael Dettweiler and Nicholas M. Katz.

[3] Mark Green, Phillip Griffiths, and Matt Kerr. Néron models and boundary components for degenerations of Hodge structure of mirror quintic type. In Curves and abelian varieties, volume 465 of Contemp. Math., pages 71-145. Amer. Math. Soc., Providence, RI, 2008.

[4] Mark Green, Phillip Griffiths, and Matt Kerr. Mumford-Tate groups and domains: their geometry and arithmetic, volume 183 of Annals of Mathematics Studies. Princeton University Press, Princeton, NJ, 2012.

[5] Roger Howe. Perspectives on invariant theory: Schur duality, multiplicity-free actions and beyond. In The Schur lectures (1992) (Tel Aviv), volume 8 of Israel Math. Conf. Proc., pages 1-182. Bar-Ilan Univ., Ramat Gan, 1995.

[6] M. Kerr and G. Pearlstein. Boundary components of Mumford-Tate domains. arXiv:1210.5301, 2012.

[7] Bertram Kostant. The principal three-dimensional subgroup and the Betti numbers of a complex simple Lie group. Amer. J. Math., 81:973-1032, 1959.

[8] C. Robles. Schubert varieties as variations of Hodge structure. arXiv:1208.5453, 2012.

E-mail address: robles@math.tamu.edu

Mathematics Department, Mail-stop 3368, Texas A\&M University, College Station, TX 77843-3368 